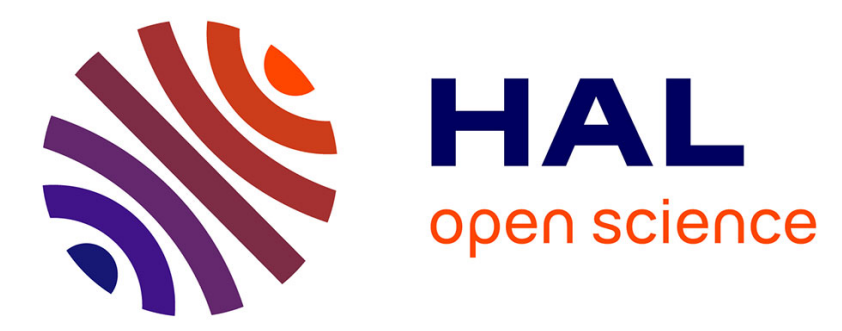

\title{
Multiresolution strategies for the modeling of composite shell structures based on the variable separation method
} Philippe Vidal, Laurent Gallimard, Olivier Polit

\section{To cite this version:}

Philippe Vidal, Laurent Gallimard, Olivier Polit. Multiresolution strategies for the modeling of composite shell structures based on the variable separation method. International Journal for Numerical Methods in Engineering, 2019, 117 (7), pp.778-799. hal-01984036

\section{HAL Id: hal-01984036 \\ https://hal.parisnanterre.fr/hal-01984036}

Submitted on 6 Feb 2019

HAL is a multi-disciplinary open access archive for the deposit and dissemination of scientific research documents, whether they are published or not. The documents may come from teaching and research institutions in France or abroad, or from public or private research centers.
L'archive ouverte pluridisciplinaire HAL, est destinée au dépôt et à la diffusion de documents scientifiques de niveau recherche, publiés ou non, émanant des établissements d'enseignement et de recherche français ou étrangers, des laboratoires publics ou privés. 


\section{Multiresolution strategies for the modeling of composite shell structures based on the variable separation method}

P. Vidal, L. Gallimard, O. Polit

\section{1 | INTRODUCTION}

Nowadays, composites are widely used in the industrial field (eg, aerospace, automotive, marine, and medical industries) due to their excellent mechanical properties, especially their high specific stiffness and strength. They are used for the manufacturing of primary structures that have to ensure the integrity of those in service. Moreover, complex geometries such as curved structures can be involved. Thus, accurate knowledge of displacements and stresses is required to design reliable systems. One way consists in considering three-dimensional (3D) modeling. However, due to the complexity of such numerical simulations, it is suitable to represent the problem as a two-dimensional (2D) model, leading to the construction of shell theories. There are two ways to define the approximation of the displacement field. A "pure shell model" can be considered, in which the displacement is associated with the local curvilinear vectors and strain and stress are deduced using differential geometry. ${ }^{1}$ Alternatively, the shell-like solid approach ${ }^{2}$ to obtain a shell finite element (FE) is widely used in commercial software, as it is more simple. In this case, the displacement vector is defined in the global Cartesian frame, and Jacobian matrix transformation is used to express strain and stress with respect to the reference frame defined on the middle surface in order to introduce the constitutive law. In this approach, differentiation is simplified, and the curvatures are not directly calculated. ${ }^{3}$ Moreover, the designer has to solve some specific problems: (i) uncertainties in 
the manufacturing process of such materials and (ii) the optimization of the structure (material and geometry) to improve the performance. These call for the resolution of numerous problems. Hence, the development of efficient computational models for the analysis of shells appears to be of major interest.

According to published research, various theories based on the FE method for composite shells have been developed. In the following, most of the mentioned works refer to the pure shell model. Thus, it is nowadays admitted that two families of models ${ }^{4}$ can be identified.

- Equivalent single-layer models (ESL). Here, the classical shell theory (CST/Koiter) and first-order shear deformation theory (Nagdhi) models are commonly used. Assumptions on the strain to derive different shell models can be found in the work of Leissa. ${ }^{5}$ The CST suffers from inaccurate results when applied to composites because both transverse shear and normal strains are neglected. The reader can refer to the works of Rao, ${ }^{6}$ Jeyachandrabose and Kirkhope, ${ }^{7}$ and Qatu and Leissa ${ }^{8}$ for discussions on shallow laminated shells. The first-order shear deformation theory is the most popular model due to the possibility of using a $\mathrm{C}^{0} \mathrm{FE}$; however, it needs shear correction factors, and the transverse normal strain is always neglected (cf the works of Reddy, ${ }^{9}$ Chakravorty et al, ${ }^{10}$ Hossain et al, ${ }^{11}$ and Asadi et $\mathrm{al}^{12}$ ). Thus, higher-order shear deformation theories have been developed to overcome these drawbacks. Different kinematics including five, ${ }^{13}$ seven, ${ }^{14,15}$ or nine parameters ${ }^{16}$ are considered. Note that various theories based on Carrera's unified formulation (CUF) are addressed in the works of Carrera et al. ${ }^{17-19}$ In the equivalent single-layer model context, a simple way to improve the estimation of the mechanical quantities consists in adding one zig-zag function (Murakami) in the expression of the displacement to introduce the slope discontinuity at the interface between two adjacent layers. It allows to describe the so-called zig-zag effect. This has been carried out in the works of Bhaskar and Varadan, ${ }^{20}$ Jing and Tzeng, ${ }^{21}$ Brank, ${ }^{22}$ and Ganapathi et al. ${ }^{23}$

- Layer-wise models (LW). Here, the expression of the mechanical quantities is written over each layer. Some of these works are based on a linear distribution of the in-plane displacements through each layer, without taking into account the transverse normal stress. The transverse displacement is assumed constant across the thickness, such as in the works of Botello et $\mathrm{al}^{24}$ and Zinno and Barbero, ${ }^{25}$ or in each layer, as in the work of Seide and Chaudhuri. ${ }^{26}$ However, this type of approach fails to predict accurate transverse stresses, unless when using special post-treatments (see, eg, the work of Zinno and Barbero ${ }^{25}$ ). Thus, higher-order approaches, taking into account the transverse normal effect, have been developed. The 3D behavior law is used. The second-, third-, and fourth-order expansions are derived in the works of Reddy, ${ }^{4}$ Basar and Ding, ${ }^{27}$ and Grigolyuk and Kulikov. ${ }^{28}$ In this framework, Kulikov and Plotnikova ${ }^{29}$ have developed the sampling surfaces method (see also the previously mentioned work ${ }^{19}$ ). In all the aforementioned works, it should be noted that the number of unknowns depends on the number of layers, affecting the performance in terms of computational cost.

As an alternative, refined models have been developed in order to improve the accuracy of ESL models, avoiding the additional computational cost of LW approaches. On the basis of physical considerations and after some algebraic transformations, the number of unknowns becomes independent of the number of layers. The deduced model can be derived from the $\mathrm{CST}^{30}$ and the higher-order shear deformation theory with a third-order theory ${ }^{31-35}$ or the sinus model. ${ }^{1}$ The number of parameters varies from 5 to 15. Thus, for all these studies, the computational cost decreases, but the transverse normal effect is introduced in none of them. This is achieved in the subsequent works of Zhen and Wanji ${ }^{36}$ and Icardi and Ferrero. ${ }^{37}$

Note also the possibility of managing different kinematics through the thickness in the framework of the CUF approach. ${ }^{38}$ The aforementioned literature deals with only some aspects of the broad research activity about composite shells. An extensive assessment of the different approaches for various theories and/or FE applications can be found in other works. ${ }^{39-47}$ For the present topics, the fundamental subject on the shear and membrane locking of the shell is not addressed here.

Over the past years, an a priori method called proper generalized decomposition (PGD) ${ }^{48-50}$ has shown interesting features in the reduction model framework. It has been successfully used in the context of the separation of coordinate variables for different types of composite structures in other works. ${ }^{51-55}$ Note that other reduction model techniques are available in open literature. A posteriori methods that consist in computing a reduced basis from snapshots using proper orthogonal decomposition or singular value decomposition are not addressed hereafter. The main goal of this work consists in assessing a multiresolution strategy based on PGD to model cylindrical composite shell structures. Classically, the present approach is based on the separation representation of the unknown displacements. In this way, the three displacements are written under the form of a sum of products of 2D polynomials of $\left(\xi^{1}, \xi^{2}\right)$ and one-dimensional (1D) polynomials of $z$. A piecewise fourth-order Lagrange polynomial is chosen for the three unknown $z$-functions. As far as the variation 
of the in-plane coordinates is concerned, a 2D eight-node quadrilateral FE is used for the three unknown $\left(\xi^{1}, \xi^{2}\right)$ functions. It should be noted that the LW unknown functions of $z$ are global for the whole shell. Finally, the deduced nonlinear problem implies the resolution of two linear problems alternatively. This process yields 2D and 1D problems in which the number of unknowns is smaller than that in a classical layer-wise approach. The interesting feature of this approach lies in the possibility of having a higher-order $z$-expansion and refining the description of the mechanical quantities through the thickness without increasing the computational cost. This is particularly suitable for modeling composite structures.

This approach can be advantageously used to perform numerous computations in which some geometric or material parameters change, with a low computational cost. It can be achieved in an optimization or a reliability process for instance. Once a first computation is carried out for a fixed set of parameters, it is possible to reuse the 2D in-plane $\left(\xi^{1}, \xi^{2}\right)$ functions previously built. Thus, only new 1D problems have to be solved for a new set of parameters. This method is an extension of the so-called preliminary stage introduced in the works of Bussy et $\mathrm{al}^{56}$ and Boisse et $\mathrm{al}^{57}$ to solve nonlinear problems with a time-space decomposition of the solution. If needed, only few 2D functions are computed to obtain an accurate solution.

We now outline the remainder of this paper. First, the shell definition, the differential geometry, and the mechanical formulation are described. Then, the principles of PGD are given. The particular assumption on the displacements yields a nonlinear problem that is solved using a fixed-point method. The classical greedy algorithm is recalled, and a multiresolution version (1D problem) is given. FE discretization is also described. Finally, numerical tests are performed on L-angle specimens that can be considered as representative structures. The variations of the stacking sequences, the geometry of the shell, and the number of layers are addressed in the multiresolution process. The efficiency of the approach is proved, and the accuracy of the results is assessed by a comparison with the layer-wise LD4 reference solution.

\section{SHELL DEFINITIONS AND DIFFERENTIAL GEOMETRY}

A shell $C$ with a middle surface $S$ and a constant thickness $e$ (see Figure 1 ) is defined by ${ }^{58}$

$$
C=\left\{M \in \mathcal{R}^{3}: \overrightarrow{O M}\left(\xi, \xi^{3}=z\right)=\vec{\Phi}(\xi)+z \vec{a}_{3} ; \xi \in \Omega ;-\frac{1}{2} e \leq z \leq \frac{1}{2} e\right\}
$$

where the middle surface can be described by a map $\vec{\Phi}$ from a parametric bidimensional domain $\Omega$ as

$$
\begin{aligned}
\vec{\Phi}: \quad \Omega \subset \mathcal{R}^{2} & \longrightarrow S \subset \mathcal{R}^{3} \\
\xi=\left(\xi^{1}, \xi^{2}\right) & \longmapsto \vec{\Phi}(\xi) .
\end{aligned}
$$

In Figure 1, the map $\vec{\Phi}$ describing the shell middle surface (in gray) and the local basis vectors are presented. The basis vectors $\vec{a}_{i}$ are defined for a point on $S$, and the basis vectors $\vec{g}_{i}$ are defined for a generic point of the shell.

For a point on the shell middle surface, the covariant basis vectors defining the tangent plane to the middle surface are usually obtained as follows:

$$
\vec{a}_{\alpha}=\vec{\Phi}\left(\xi^{1}, \xi^{2}\right)_{, \alpha} \quad ; \quad \vec{a}_{3}=\frac{\vec{a}_{1} \times \vec{a}_{2}}{\left\|\vec{a}_{1} \times \vec{a}_{2}\right\|},
$$

where $\vec{a}_{3}$ is the unit normal vector to the surface $S$ (see Figure 1). In Equation (2) and further on, Latin indices $i, j, \ldots$ take their values in the set $\{1,2,3\}$, whereas Greek indices $\alpha, \beta, \ldots$ take their values in the set $\{1,2\}$. The summation

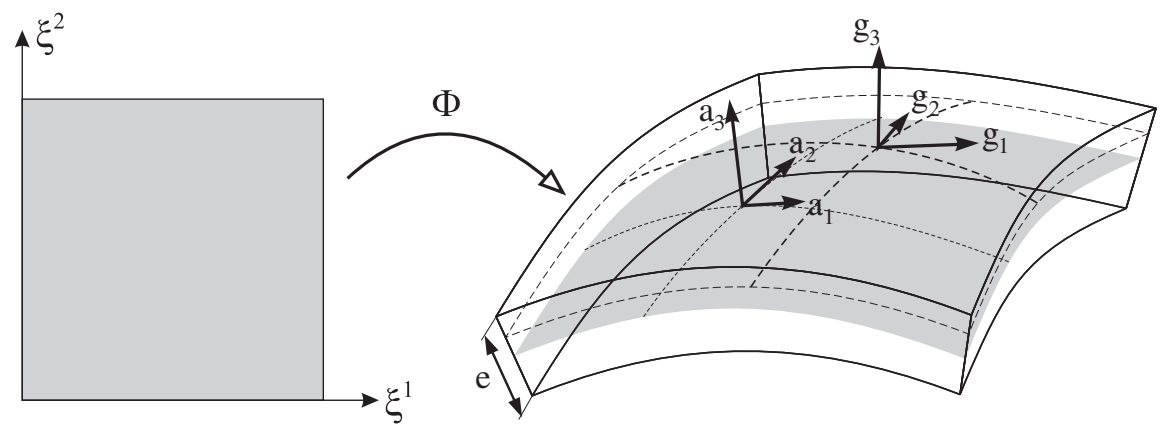

FIGURE 1 The map $\vec{\Phi}$ and the local basis vectors $\vec{a}_{i}$ and $\vec{g}_{i}$ for a shell panel 
convention on repeated indices is used, and the partial derivative is denoted by ()$_{, \alpha}$. A shell is characterized by the first fundamental form $a_{\alpha \beta}$ and the second fundamental form $b_{\alpha \beta}$. Their covariant, contravariant, and mixed-form definitions are given by

$$
a_{\alpha \beta}=\vec{a}_{\alpha} \cdot \vec{a}_{\beta} \quad a^{\alpha \beta}=\vec{a}^{\alpha} \cdot \vec{a}^{\beta} \quad b_{\alpha \beta}=\vec{a}_{\alpha, \beta} \cdot \vec{a}_{3} \quad b^{\beta}{ }_{\alpha}=\vec{a}^{\beta} \cdot \vec{a}_{3, \alpha} .
$$

For a generic point of the shell, covariant basis vectors must be defined, and we have

$$
\vec{g}_{\alpha}=\overrightarrow{O M}(\xi, z)_{, \alpha}=\left(\delta_{\alpha}^{\beta}-z b_{\alpha}^{\beta}\right) \vec{a}_{\beta}=\mu_{\alpha}^{\beta}(z) \vec{a}_{\beta} \quad \text { and } \quad \vec{g}_{3}=\vec{a}_{3},
$$

where $\delta_{\alpha}^{\beta}$ is the Kronecker symbol and $b_{\alpha}^{\beta}$ is the mixed form of the second fundamental form. This basis $\vec{g}_{i}$, illustrated in Figure 1, must be used to define quantities for any point of the shell. The form $\mu_{\alpha}^{\beta}(z)$ introduced in Equation (4) defines the transport from the shell middle surface to any point of the shell and is associated with the curvature variation along the thickness direction $z$ of the shell. The inverse tensor of the mixed tensor $\mu_{\alpha}^{\beta}$ is denoted $m_{\alpha}^{\beta}$ and is defined as

$$
m_{\alpha}^{\beta}=\left(\mu^{-1}\right)_{\alpha}^{\beta}=\frac{1}{\mu}\left\{\delta_{\alpha}^{\beta}+z\left(b_{\alpha}^{\beta}-2 H \delta_{\alpha}^{\beta}\right)\right\},
$$

where we have introduced the determinant of the mixed tensor $\mu=\operatorname{det}\left(\mu_{\alpha}^{\beta}\right)=1-2 H \xi^{3}+\left(\xi^{3}\right)^{2} K$ and the invariants of the second fundamental form $H=\frac{1}{2} \operatorname{tr}\left(b_{\alpha}^{\beta}\right)$ and $K=\operatorname{det}\left(b^{\beta}{ }_{\alpha}\right)$. Finally, the surface element $d S$ and the volume element $d C$ are given by

$$
d S=\sqrt{a} d \xi^{1} d \xi^{2} \quad d C=\mu d S d z
$$

where $a$ is the determinant of the first fundamental form $a_{\alpha \beta}$. The geometry of a shell can also be defined using a contravariant or mixed forms. Furthermore, covariant and contravariant differentiation involving Christoffel symbols is not detailed here, and readers can refer to the book of Bernadou. ${ }^{58}$

\section{3 | REFERENCE PROBLEM DESCRIPTION}

\subsection{The definition of the strain field}

For geometrically linear elastic analysis, the components of the strain tensor $\varepsilon_{i j}$ expressed in the contravariant basis $\vec{a}^{i}$ are obtained through covariant differentiation, denoted |, as follows:

$$
\begin{aligned}
\varepsilon & =\varepsilon_{i j}\left(\vec{a}^{i} \otimes \vec{a}^{j}\right) \text { with } \\
2 \varepsilon_{\gamma \lambda} & =m^{\beta}{ }_{\lambda}\left(u_{\gamma \mid \beta}-b_{\gamma \beta} u_{3}\right)+m_{\gamma}^{\alpha}{ }_{\gamma}\left(u_{\lambda \mid \alpha}-b_{\lambda \alpha} u_{3}\right) \\
2 \varepsilon_{\gamma 3} & =u_{\gamma \mid 3}+m^{\alpha}{ }_{\gamma}\left(u_{3, \alpha}+b_{\alpha}^{\lambda} u_{\lambda}\right) \\
\varepsilon_{33} & =u_{3,3} .
\end{aligned}
$$

The mixed tensor $m_{\lambda}^{\beta}$ carries out the transport from any point of the shell to the shell middle surface, that is, from $\vec{g}^{i}$ to $\vec{a}^{i}$.

\section{2 | Constitutive relation}

The stress tensor is obtained from the strain tensor using the constitutive equations. For this purpose, all these tensors must be referred to the covariant and contravariant basis vectors, $\vec{a}_{i}$ and $\vec{a}^{i}$, respectively, associated with the middle surface of the shell. In case of laminated shells composed of orthotropic plies, this reference frame ensures to consistently take into account the different material orientations of the layers. The tensorial relation is

$$
\sigma^{i j}=C^{i j k l} \varepsilon_{k l} \text { with } \sigma^{i j}\left(\vec{a}_{i} \otimes \vec{a}_{j}\right), \quad C^{i j k l}\left(\vec{a}_{i} \otimes \vec{a}_{j} \otimes \vec{a}_{k} \otimes \vec{a}_{l}\right), \quad \varepsilon_{k l}\left(\vec{a}^{k} \otimes \vec{a}^{l}\right) .
$$

It should be noted that the stress tensor is defined in the covariant reference frame, whereas the strain components are defined in the contravariant frame. If the frame is assumed to be orthonormal, then the covariant and contravariant 
components are equal, that is, the superscript and subscript are interchangeable. With this assumption and for a shell made of $N C$ perfectly bonded orthotropic layers, the 3D constitutive law of the $k$ th layer is expressed in matrix notation by

$$
\left[\begin{array}{c}
\sigma_{11}{ }^{(k)}(\xi, z) \\
\sigma_{22}{ }^{(k)}(\xi, z) \\
\sigma_{33}{ }^{(k)}(\xi, z) \\
\sigma_{23}{ }^{(k)}(\xi, z) \\
\sigma_{13}{ }^{(k)}(\xi, z) \\
\sigma_{12}{ }^{(k)}(\xi, z)
\end{array}\right]=\left[\begin{array}{cccccc}
C_{11}{ }^{(k)} & C_{12}{ }^{(k)} & C_{13}{ }^{(k)} & 0 & 0 & 0 \\
& C_{22}{ }^{(k)} & C_{23}{ }^{(k)} & 0 & 0 & 0 \\
& & C_{33}{ }^{(k)} & 0 & 0 & 0 \\
& & & C_{44}{ }^{(k)} & 0 & 0 \\
& & & & C_{55}{ }^{(k)} & 0 \\
& & & & & C_{66}{ }^{(k)}
\end{array}\right]\left[\begin{array}{c}
\varepsilon_{11}{ }^{(k)}(\xi, z) \\
\varepsilon_{22}{ }^{(k)}(\xi, z) \\
\varepsilon_{33}{ }^{(k)}(\xi, z) \\
\gamma_{23}{ }^{(k)}(\xi, z) \\
\gamma_{13}{ }^{(k)}(\xi, z) \\
\gamma_{12}{ }^{(k)}(\xi, z)
\end{array}\right],
$$

ie, $\boldsymbol{\sigma}^{(k)}=\mathbf{C}^{(k)} \boldsymbol{\varepsilon}^{(k)}$,

where we denote $C_{i j}^{(k)}$ as the 3D stiffness coefficients of layer $k$, stress vector $\sigma$, and strain vector $\varepsilon$.

\subsection{The weak form of the boundary value problem}

The shell is submitted to a surface force density $\mathbf{t}$ defined over a subset $\partial \mathcal{C}_{F}$ of the boundary. We assume that a prescribed displacement $\mathbf{u}=\mathbf{u}_{d}$ is imposed on $\Gamma_{D}=\partial \mathcal{C}-\partial \mathcal{C}_{F}$. The mechanical problem is based on the variational principle, which is given as follows: find $\mathbf{u}(M) \in U$ (space of admissible displacements) such that

$$
a(\mathbf{u}, \delta \mathbf{u})=b(\delta \mathbf{u}), \quad \forall \delta \mathbf{u} \in \delta U
$$

with

$$
\begin{aligned}
a(\mathbf{u}, \delta \mathbf{u}) & =\iiint_{\mathcal{C}} \delta \boldsymbol{\varepsilon}^{T} \boldsymbol{\sigma} d \mathcal{C} \\
b(\delta \mathbf{u}) & =\iint_{\partial C_{F}} \delta \mathbf{u} \cdot \mathbf{t} d \partial \mathcal{C},
\end{aligned}
$$

where $U$ is the space of admissible displacements, ie, $U=\left\{\mathbf{u} \in\left(H^{1}(\mathcal{V})\right)^{3} / \mathbf{u}=\mathbf{u}_{d}\right.$ on $\left.\Gamma_{D}\right\}$. We have also $\delta U=\left\{\mathbf{u} \in\left(H^{1}(\mathcal{V})\right)^{3} / \mathbf{u}=0\right.$ on $\left.\Gamma_{D}\right\}$.

For the present work, $\partial \mathcal{C}_{F}$ is considered as the top or bottom surface of the shell, that is, $z=z_{F}$ with $z_{F}= \pm e / 2$. For the sake of clarity, the body forces are neglected in the developments.

\section{APPLICATION OF PGD TO A CYLINDRICAL SHELL}

In this section, the application of PGD is developed in order to carry out numerous shell analyses. This work is an extension of the previous studies on beam, plate, and shell structures..$^{53-55}$

\section{1 | Cylindrical geometry}

A cylindrical shell (see Figure 2) is described using the following map:

$$
\left\{\begin{array}{l}
x_{1}\left(\xi^{1}, \xi^{2}\right)=R \cos \left(\frac{\xi^{1}}{R}\right) \\
x_{2}\left(\xi^{1}, \xi^{2}\right)=R \sin \left(\frac{\xi^{1}}{R}\right) \\
x_{3}\left(\xi^{1}, \xi^{2}\right)=\xi^{2} .
\end{array}\right.
$$

Following Equation (3), the nonzero terms for the covariant and mixed forms are

$$
a_{11}=a_{22}=1 \quad b_{11}=b^{1}{ }_{1}=-\frac{1}{R} \quad \mu^{1}{ }_{1}=1+\frac{z}{R} \quad m_{1}{ }_{1}=\left(1+\frac{z}{R}\right)^{-1}
$$

and $\mu=1+\frac{z}{R}$. 


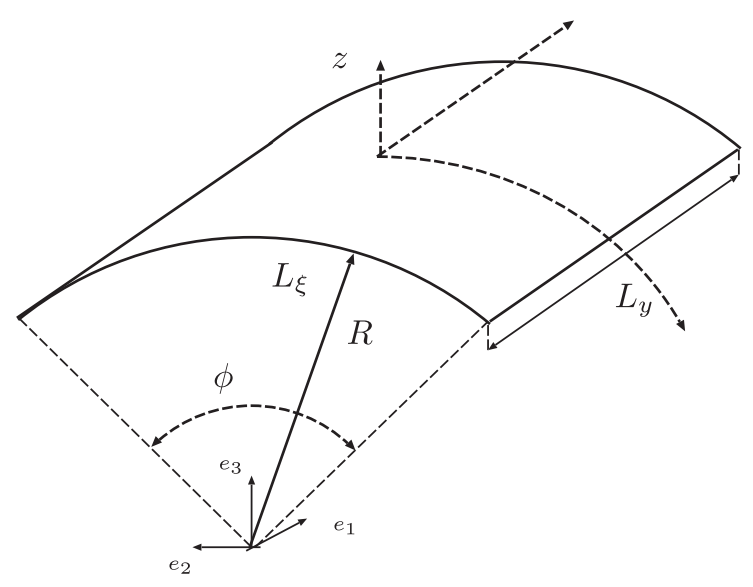

FIGURE 2 Middle surface of the cylindrical shell

\section{2 | The displacement}

Let us denote $\left(u_{1}\left(\xi^{1}, \xi^{2}, \xi^{3}=z\right), u_{2}\left(\xi^{1}, \xi^{2}, \xi^{3}=z\right), u_{3}\left(\xi^{1}, \xi^{2}, \xi^{3}=z\right)\right)$ as the curvilinear components of the displacement field associated with the contravariant basis vectors $\vec{a}^{i}$. Let $\Omega$ and $\Omega_{z}$ be the bidimensional domain associated with the mid-surface of the shell (see Equation (1)) and the unidimensional domain associated with the normal fiber, respectively. This displacement solution is constructed as the sum of $N$ products of functions of in-plane coordinates and the transverse coordinate ( $N \in \mathbb{N}$ is the order of the representation), ie,

$$
\mathbf{u}=\left[\begin{array}{l}
u_{1}\left(\xi, \xi^{3}=z\right) \\
u_{2}\left(\xi, \xi^{3}=z\right) \\
u_{3}\left(\xi, \xi^{3}=z\right)
\end{array}\right]=\sum_{i=1}^{N}\left[\begin{array}{l}
f_{1}^{i}(z) v_{1}^{i}(\xi) \\
f_{2}^{i}(z) v_{2}^{i}(\xi) \\
f_{3}^{i}(z) v_{3}^{i}(\xi)
\end{array}\right]=\sum_{i=1}^{N}\left[\begin{array}{l}
f_{1}^{i}(z) \\
f_{2}^{i}(z) \\
f_{3}^{i}(z)
\end{array}\right] \circ\left[\begin{array}{c}
v_{1}^{i}(\xi) \\
v_{2}^{i}(\xi) \\
v_{3}^{i}(\xi)
\end{array}\right]
$$

where $\left(f_{1}^{j}, f_{2}^{j}, f_{3}^{j}\right)$ are defined in $\Omega_{z}$ and $\left(v_{1}^{j}, v_{2}^{j}, v_{3}^{j}\right)$ are defined in $\Omega$. The “o" operator is Hadamard's element-wise product. In this paper, a classical eight-node FE approximation is used in $\Omega$, and an LW description is chosen in $\Omega_{z}$ as it is particularly suitable for modeling a composite structure.

\subsection{The strain field for the cylindrical composite structure}

The strain field in Equation (7) is free of any approximated shell kinematics. These strain components are simplified using Equation (12), and we recover the following relations:

$$
\begin{aligned}
& \varepsilon_{11}=\left(1+\frac{z}{R}\right)^{-1}\left(u_{1,1}+\frac{1}{R} u_{3}\right) \\
& \varepsilon_{22}=u_{2,2} \\
& \varepsilon_{33}=u_{3,3} \\
& \gamma_{23}=u_{2,3}+u_{3,2} \\
& \gamma_{13}=u_{1,3}+\left(1+\frac{z}{R}\right)^{-1}\left(u_{3,1}-\frac{1}{R} u_{1}\right) \\
& \gamma_{12}=u_{1,2}+\left(1+\frac{z}{R}\right)^{-1} u_{2,1},
\end{aligned}
$$

where the covariant derivative becomes a classical derivative for the case of a cylindrical shell. 
Equation (13) must be introduced at this level in order to obtain the compatible strain expansion along the normal coordinate $z$ of the shell. Hence, we obtain

$$
\varepsilon(u)=\sum_{j=1}^{N}\left[\begin{array}{c}
\mu^{-1}\left(f_{1}^{j} v_{1,1}^{j}+\frac{1}{R} f_{3}^{j} v_{3}^{j}\right) \\
f_{2}^{j} v_{2,2}^{j} \\
\left(f_{3}^{j}\right)^{\prime} v_{3}^{j} \\
\left(f_{2}^{j}\right)^{\prime} v_{2}^{j}+f_{3}^{j} v_{3,2}^{j} \\
\left(f_{1}^{j}\right)^{\prime} v_{1}^{j}+\mu^{-1}\left(f_{3}^{j} v_{3,1}^{j}-\frac{1}{R} f_{1}^{j} v_{1}^{j}\right) \\
f_{1}^{j} v_{1,2}^{j}+\mu^{-1} f_{2}^{j} v_{2,1}^{j}
\end{array}\right],
$$

where the prime stands for the classical derivative $\left(f_{i}^{\prime}=\frac{d f_{i}}{d z}\right),()_{, \alpha}$ stands for the partial derivative, and $\mu=1+\frac{z}{R}$.

\subsection{The problem to be solved}

The resolution of Equation (10) is based on a greedy algorithm. If we assume that the first $m$ functions have been already computed, the trial function for the iteration $m+1$ is written as

$$
\mathbf{u}^{m+1}=\mathbf{u}^{m}+\left[\begin{array}{l}
f_{1} v_{1} \\
f_{2} v_{2} \\
f_{3} v_{3}
\end{array}\right]=\mathbf{u}^{m}+\mathbf{f} \circ \mathbf{v},
$$

where $\left(v_{1}, v_{2}, v_{3}\right)$ and $\left(f_{1}, f_{2}, f_{3}\right)$ are the functions to be computed and $\mathbf{u}^{m}$ is the associated known set at iteration $m$ defined by

$$
\mathbf{u}^{m}=\sum_{i=1}^{m}\left[\begin{array}{l}
f_{1}^{i} v_{1}^{i} \\
f_{2}^{i} v_{2}^{i} \\
f_{3}^{i} v_{3}^{i}
\end{array}\right] .
$$

The test functions are

$$
\delta(\mathbf{f} \circ \mathbf{v})=\delta \mathbf{f} \circ \mathbf{v}+\delta \mathbf{v} \circ \mathbf{f}
$$

with

$$
\mathbf{v}=\left[\begin{array}{l}
v_{1} \\
v_{2} \\
v_{3}
\end{array}\right] \mathbf{f}=\left[\begin{array}{l}
f_{1} \\
f_{2} \\
f_{3}
\end{array}\right] .
$$

Introducing the test function defined by Equation (18) and the trial function defined by Equation (16) into the weak form given by Equation (10), the two following equations to be solved can be deduced:

- for the test function $\delta \mathbf{f}$, we have

$$
a(\mathbf{v} \circ \mathbf{f}, \mathbf{v} \circ \delta \mathbf{f})=b(\mathbf{v} \circ \delta \mathbf{f})-a\left(\mathbf{u}^{m}, \mathbf{v} \circ \delta \mathbf{f}\right) \quad \forall \delta \mathbf{f} ;
$$

- for the test function $\delta \mathbf{v}$, we have

$$
a(\mathbf{f} \circ \mathbf{v}, \mathbf{f} \circ \delta \mathbf{v})=b(\mathbf{f} \circ \delta \mathbf{v})-a\left(\mathbf{u}^{m}, \mathbf{f} \circ \delta \mathbf{v}\right) \quad \forall \delta \mathbf{v} .
$$

This coupled nonlinear problem is solved using a classical strategy based on a fixed-point method. For each problem, only an unknown 1D or 2D function has to be found. Hence, the approach leads to the process given in Algorithm 1. The fixed-point algorithm is stopped when the distance between two consecutive terms is sufficiently small (cf Vidal et $\mathrm{al}^{54}$ ). 


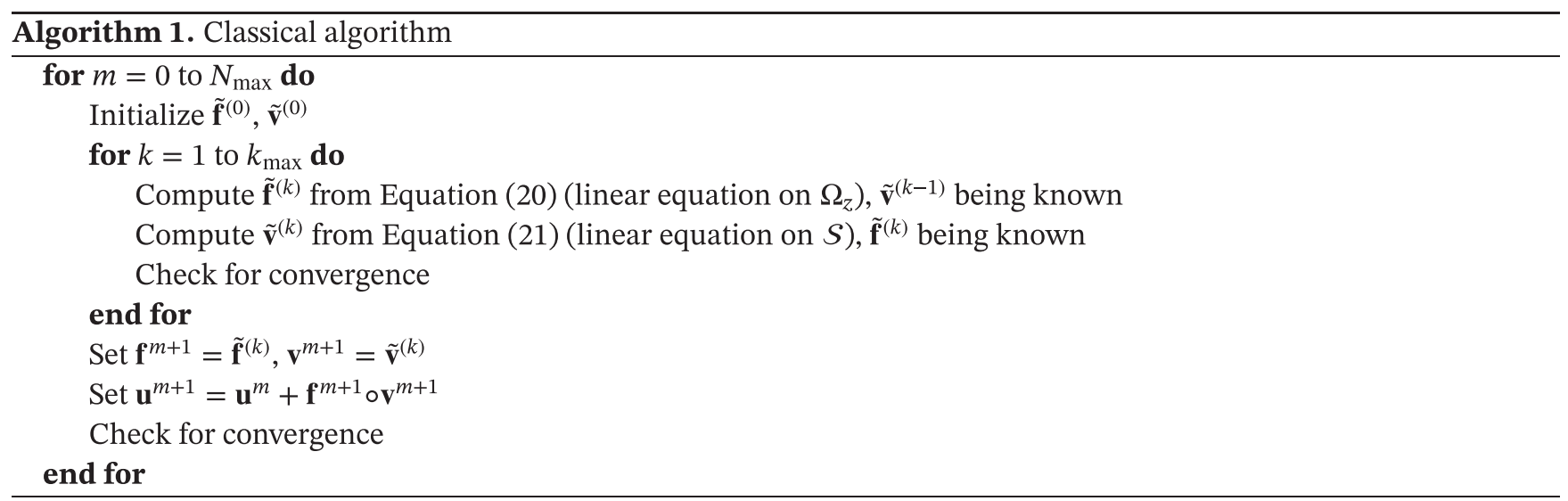

\subsection{Multiresolution algorithm with a preliminary stage}

In this section, a multiresolution algorithm is given, using the advantages of the present variable separation method. The approach described here can be considered as an extension of the so-called preliminary stage introduced in the works of Bussy et $\mathrm{al}^{56}$ and Boisse et $\mathrm{l}^{57}$ to improve the convergence rate of the LArge Time Increment (LATIN) method. Once some couples (at least two) are built, the process consists in updating only all the $1 \mathrm{D} z$-functions $f_{i}^{j}(z)$, with the basis functions $v_{i}^{j}(\xi)$ being known. Thus, assuming that $N_{1}$ couples are built, the problem to be solved can be written as

$$
a\left(\sum_{i=1}^{N_{1}} \mathbf{v}^{i} \circ \mathbf{f}^{i}, \sum_{i=1}^{N_{1}} \mathbf{v}^{i} \circ \delta \mathbf{f}^{i}\right)=b\left(\sum_{i=1}^{N_{1}} \mathbf{v}^{i} \circ \delta \mathbf{f}^{i}\right) \quad \forall \delta \mathbf{f}^{i} .
$$

The associated multiresolution algorithm is given in Algorithm 2. The first step consists in building $N_{1}$ couples for an initial set of parameters. Then, these $\xi$-functions $\mathbf{v}^{p}$ are reused in the following calculations involving new sets of parameters. Thus, only the $z$-functions are updated. The associated problem is $1 \mathrm{D}$. If the residual error remains high, only few new couples are computed to achieve the convergence in the so-called enrichment stage.

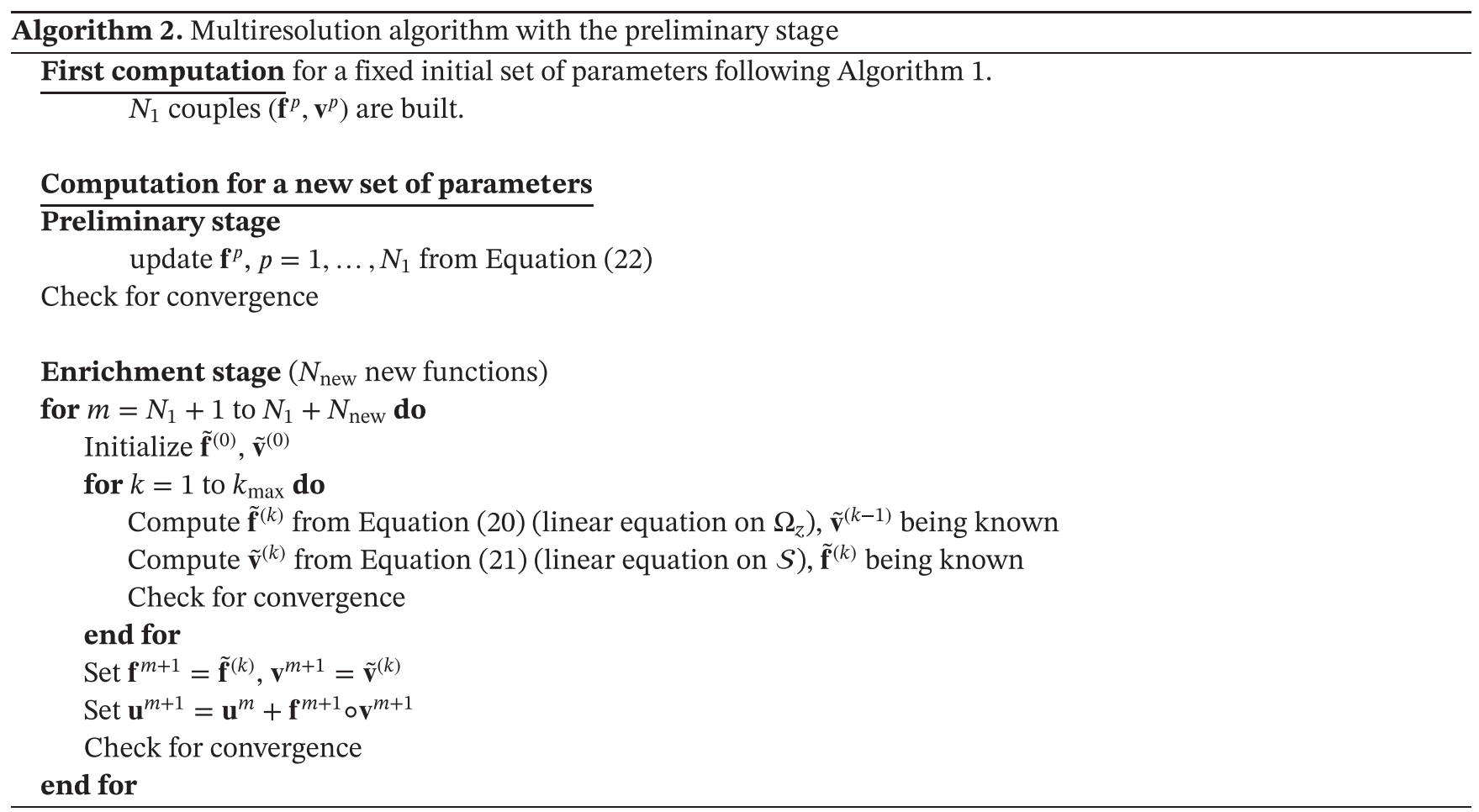


Remark 1. The convergence rate of the classical Algorithm 1 can be improved by introducing the preliminary stage at each iteration of the algorithm to update the $1 \mathrm{D} z$-functions. The new associated algorithm is given in Algorithm 3.

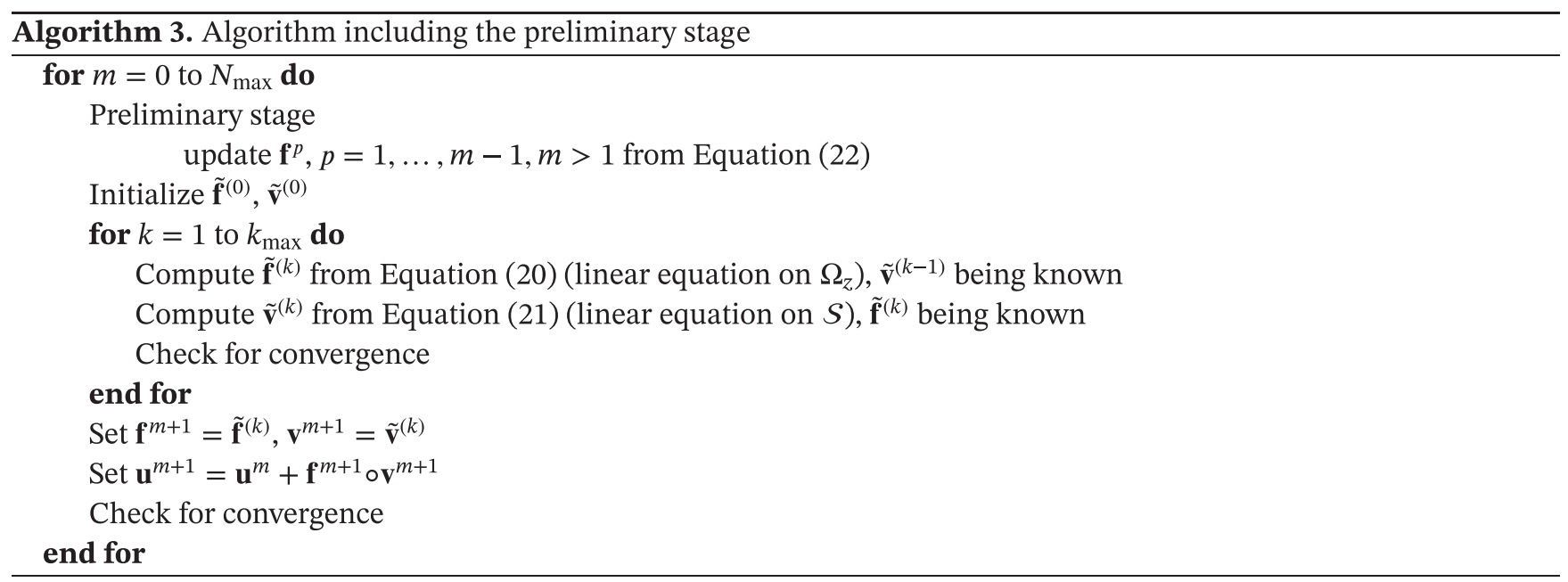

\section{6 | FE discretization}

To build the shell FE approximation, a discrete representation of the functions $(\mathbf{v}, \mathbf{f})$ must be introduced. We use a classical FE approximation in $\Omega$ and a polynomial expansion in $\Omega_{z}$. The elementary vector of degrees of freedom (dofs) associated with one element $\Omega_{e}$ of the mesh in $\Omega$ is denoted $\mathbf{q}_{e}^{v}$. The vector of dofs associated with the polynomial expansion in $\Omega_{z}$ is denoted $\mathbf{q}^{f}$. The displacement fields and the strain fields are determined from the values of $\mathbf{q}_{e}^{v}$ and $\mathbf{q}_{e}^{f}$ by

$$
\begin{aligned}
\mathbf{v}_{e}=\mathbf{N}_{\xi} \mathbf{q}_{e}^{v}, & \mathcal{E}_{v}^{e}=\mathbf{B}_{\xi} \mathbf{q}_{e}^{v}, \\
\mathbf{f}_{e}=\mathbf{N}_{z} \mathbf{q}_{e}^{f}, & \mathcal{E}_{f}^{e}=\mathbf{B}_{z} \mathbf{q}_{e}^{f},
\end{aligned}
$$

where

$$
\mathcal{E}_{v}^{e^{T}}=\left[\begin{array}{lllllllll}
v_{1} & v_{1,1} & v_{1,2} & v_{2} & v_{2,1} & v_{2,2} & v_{3} & v_{3,1} & v_{3,2}
\end{array}\right]
$$

and

$$
\mathcal{E}_{f}^{e^{T}}=\left[\begin{array}{llllll}
f_{1} & f_{1}^{\prime} & f_{2} & f_{2}^{\prime} & f_{3} & f_{3}^{\prime}
\end{array}\right]
$$

The matrices $\mathbf{N}_{\xi}, \mathbf{B}_{\xi}, \mathbf{N}_{z}$, and $\mathbf{B}_{z}$ contain the interpolation functions, their derivatives, and the Jacobian components.

\section{7 | FE problem to be solved on $\Omega$}

For the sake of simplicity, the function $\tilde{\mathbf{f}}^{(k)}$, which is assumed to be known, will be denoted $\tilde{\mathbf{f}}$, and the function $\tilde{\mathbf{v}}^{(k)}$ to be computed will be denoted $\mathbf{v}$. The strain in Equation (21) is defined in matrix notations as

$$
\varepsilon(\tilde{f} \circ v)=\Sigma_{z}(\tilde{f}) \mathcal{E}_{v}
$$

with

$$
\Sigma_{\mathbf{z}}(\tilde{f})=\left[\begin{array}{ccccccccc}
0 & \tilde{f}_{1} / \mu & 0 & 0 & 0 & 0 & \tilde{f}_{3} /(\mu R) & 0 & 0 \\
0 & 0 & 0 & 0 & 0 & \tilde{f}_{2} & 0 & 0 & 0 \\
0 & 0 & 0 & 0 & 0 & 0 & \tilde{f}_{3}^{\prime} & 0 & 0 \\
0 & 0 & 0 & \tilde{f}_{2}^{\prime} & 0 & 0 & 0 & 0 & \tilde{f}_{3} \\
\tilde{f}_{1}^{\prime}-\tilde{f}_{1} /(\mu R) & 0 & 0 & 0 & 0 & 0 & 0 & \tilde{f}_{3} / \mu & 0 \\
0 & 0 & \tilde{f}_{1} & 0 & \tilde{f}_{2} / \mu & 0 & 0 & 0 & 0
\end{array}\right] .
$$

The variational problem defined on $\Omega$ from Equations (6) and (21) is

$$
\int_{\Omega} \delta \mathcal{E}_{\mathbf{v}}{ }^{T} \mathbf{k}_{z}(\tilde{f}) \mathcal{E}_{\mathbf{v}} \sqrt{a} \underbrace{d \Omega}_{d \xi^{1} d \xi^{2}}=\int_{\Omega} \delta \mathbf{v}^{T} \mathbf{t}_{z}(\tilde{f}) \sqrt{a} d \Omega-\int_{\Omega} \delta \mathcal{E}_{\mathbf{v}}{ }^{T} \boldsymbol{\sigma}_{z}\left(\tilde{f}, \mathbf{u}^{m}\right) \sqrt{a} d \Omega
$$


with

$$
\begin{aligned}
\mathbf{k}_{z}(\tilde{f}) & =\int_{\Omega_{z}} \Sigma_{\mathbf{z}}(\tilde{f})^{T} \mathbf{C} \Sigma_{\mathbf{z}}(\tilde{f}) \mu d z \\
\mathbf{t}_{z}(\tilde{f}) & =\left.\tilde{\mathbf{f}} \circ \mathbf{t} \mu\right|_{z=z_{F}} \\
\sigma_{z}\left(\tilde{f}, \mathbf{u}^{m}\right) & =\int_{\Omega_{z}} \Sigma_{\mathbf{z}}(\tilde{f})^{T} \mathbf{C} \varepsilon\left(\mathbf{u}^{m}\right) \mu d z .
\end{aligned}
$$

Note that the calculation of Equations (27) and (29) is performed using an analytical integration.

The introduction of the FE approximation given by Equation (23) in the variational Equation (26) leads to the linear system

$$
\mathbf{K}_{z}(\tilde{f}) \mathbf{q}^{v}=\mathcal{R}_{v}\left(\tilde{f}, \mathbf{u}^{m}\right),
$$

where

- $\mathbf{q}^{v}$ is the vector of the nodal displacements in the contravariant basis,

- $\mathbf{K}_{z}(\tilde{f})$ is the stiffness matrix obtained by summing up the elements' stiffness matrices $\mathbf{K}_{z}^{e}(\tilde{f})=\int_{\Omega_{e}} \mathbf{B}_{\xi}^{T} \mathbf{k}_{z}(\tilde{f}) \mathbf{B}_{\xi} \sqrt{a} d \Omega_{e}$, and

- $\mathcal{R}_{v}\left(\tilde{f}, \mathbf{u}^{m}\right)$ is the equilibrium residual obtained by summing up the elements' residual load vectors $\mathcal{R}_{v}^{e}\left(\tilde{f}, \mathbf{u}^{m}\right)=\int_{\Omega_{e}} \mathbf{N}_{\xi}^{T} \mathbf{t}_{z}(\tilde{f}) \sqrt{a} d \Omega_{e}-\int_{\Omega_{e}} \mathbf{B}_{\xi}^{T} \boldsymbol{\sigma}_{z}\left(\tilde{f}, \mathbf{u}^{m}\right) \sqrt{a} d \Omega_{e}$.

\subsection{FE problem to be solved on $\Omega_{z}$}

For the sake of simplicity, the function $\tilde{\mathbf{v}}^{(k-1)}$, which is assumed to be known, will be denoted $\tilde{\mathbf{v}}$, and the function $\tilde{\mathbf{f}}^{(k)}$ to be computed will be denoted $\mathbf{f}$. The strain in Equation (20) is defined in matrix notations as

$$
\varepsilon(\tilde{v} \circ f)=\Sigma_{\xi}(\tilde{v}) \mathcal{E}_{\mathbf{f}}
$$

with

$$
\Sigma_{\xi}(\tilde{v})=\left[\begin{array}{cccccc}
\tilde{v}_{1,1} / \mu & 0 & 0 & 0 & \tilde{v}_{3} /(\mu R) & 0 \\
0 & 0 & \tilde{v}_{2,2} & 0 & 0 & 0 \\
0 & 0 & 0 & 0 & 0 & \tilde{v}_{3} \\
0 & 0 & 0 & \tilde{v}_{2} & \tilde{v}_{3,2} & 0 \\
-\tilde{v}_{1} /(\mu R) & \tilde{v}_{1} & 0 & 0 & \tilde{v}_{3,1} / \mu & 0 \\
\tilde{v}_{1,2} & 0 & \tilde{v}_{2,1} / \mu & 0 & 0 & 0
\end{array}\right] .
$$

The variational problem defined on $\Omega_{z}$ from Equation (20) is

$$
\int_{\Omega_{z}} \delta \mathcal{E}_{\mathbf{f}}^{T} \mathbf{k}_{\xi}(\tilde{v}) \mathcal{E}_{\mathbf{f}} \mu d z=\left.\delta \mathbf{f}^{T} \mathbf{t}_{\xi}(\tilde{v}) \mu\right|_{z=z_{F}}-\int_{\Omega_{z}} \delta \mathcal{E}_{\mathbf{f}}^{T} \boldsymbol{\sigma}_{\xi}\left(\tilde{v}, \mathbf{u}^{m}\right) \mu d z
$$

with

$$
\begin{aligned}
\mathbf{k}_{\xi}(\tilde{\mathcal{V}}) & =\int_{\Omega} \Sigma_{\xi}(\tilde{\mathcal{v}})^{T} \mathbf{C} \Sigma_{\xi}(\tilde{v}) \sqrt{a} d \Omega \\
\mathbf{t}_{\xi}(\tilde{\mathcal{v}}) & =\int_{\Omega} \tilde{\mathbf{v}} \circ \mathbf{t} \sqrt{a} d \Omega \\
\boldsymbol{\sigma}_{\xi}\left(\tilde{v}, \mathbf{u}^{m}\right) & =\int_{\Omega} \Sigma_{\xi}(\tilde{\mathcal{v}})^{T} \mathbf{C} \varepsilon\left(\mathbf{u}^{m}\right) \sqrt{a} d \Omega .
\end{aligned}
$$

The introduction of the FE discretization given by Equation (23) in the variational Equation (33) leads to the linear system

$$
\mathbf{K}_{\xi}(\tilde{v}) \mathbf{q}^{f}=\mathcal{R}_{f}\left(\tilde{v}, \mathbf{u}^{m}\right),
$$

where $\mathbf{q}^{f}$ is the vector of dofs associated with the polynomial expansion in $\Omega_{z}, \mathbf{K}_{\xi}(\tilde{v})$ is a stiffness matrix defined by Equation (38), and $\mathcal{R}_{f}\left(\tilde{v}, \mathbf{u}^{m}\right)$ is an equilibrium residual defined by Equation (39), ie,

$$
\mathbf{K}_{\xi}(\tilde{v})=\int_{\Omega_{z}} \mathbf{B}_{z}^{T} \mathbf{k}_{\xi}(\tilde{v}) \mathbf{B}_{z} \mu d z
$$




$$
\mathcal{R}_{f}\left(\tilde{v}, \mathbf{u}^{m}\right)=\left.\mathbf{N}_{z}^{T} \mathbf{t}_{\xi}(\tilde{v}) \mu\right|_{z=z_{F}}-\int_{\Omega_{z}} \mathbf{B}_{z}^{T} \boldsymbol{\sigma}_{\xi}\left(\tilde{v}, \mathbf{u}^{m}\right) \mu d z
$$

Remark 2. For the preliminary stage, the same type of computation has to be performed. The system to be solved can be written under the following form:

$$
\mathbf{K}_{\mathrm{PS}}\left(v^{1}, \ldots, v^{m-1}\right) \mathbf{q}^{f^{1 m-1}}=\mathcal{R}_{f}\left(v^{1}, \ldots, v^{m-1}\right),
$$

where

- $\mathbf{K}_{\mathrm{PS}}\left(v^{1}, \ldots, v^{m-1}\right)$ is the assembly of matrices $\mathbf{K}_{\xi}\left(v^{i}, v^{j}\right)=\int_{\Omega_{z}} \mathbf{B}_{z}^{T} \mathbf{k}_{\xi}\left(v^{i}, v^{j}\right) \mathbf{B}_{z} \mu d z$ with $\mathbf{k}_{\xi}\left(v^{i}, v^{j}\right)=\int_{\Omega} \Sigma_{\xi}\left(v^{i}\right)^{T} \mathbf{C} \Sigma_{\xi}\left(v^{j}\right) \sqrt{a} d \Omega$,

- $\mathcal{R}_{f}\left(v^{1}, \ldots, v^{m-1}\right)$ is written with the submatrices $\left.\mathbf{N}_{z}^{T} \mathbf{t}_{\xi}\left(v^{i}\right) \mu\right|_{z=z_{F}}$, and

- $\mathbf{q}^{f^{1 m-1} T}=\left[\mathbf{q}^{f^{1 T}} \cdots \mathbf{q}^{f^{m-1} T}\right]$.

In fact, the computational cost of this stage is mainly due to the calculation of the integrals. The order of magnitude is proportional to $N_{z} \times N_{x} \times N_{y} \times N_{\text {couple }}$, where $N_{x}, N_{y}$ are the number of elements in the $\xi_{1}, \xi_{2}$ directions, respectively. $N_{z}$ is the number of numerical layers (number of elements in $\Omega_{z}$ ), and $N_{\text {couple }}$ is the number of couples built in the PGD process. Nevertheless, as shown in the work of Vidal et al, ${ }^{54}$ the estimation of the total computational time is proportional to $N_{x}^{3} \times N_{y} \times N_{\text {couple }}$ (for $N_{x}>N_{y}$, and a high value of $N_{x} \times N_{y}, N_{z}$ ). Thus, the additional cost of the preliminary stage remains low.

\section{5 | NUMERICAL RESULTS}

In this section, an eight-node quadrilateral FE based on the Serendipity interpolation functions is used for the unknowns depending on the in-plane coordinates. The geometry of the shell is approximated by this classical FE in the parametric space. The geometrical transformation is based on an explicit map $\vec{\Phi}$. A Gaussian numerical integration with $3 \times 3$ points is used to calculate the elementary matrices.

In the framework of multiparametric processes, it is required to reduce significantly the cost of the analyses, which can be numerous. Hereafter, as explained in Section 4.5, we take advantage of the possibility offered by the present method to reuse the basis of space $\xi$ functions built during the first resolution. Indeed, once a first analysis has been carried out for a set of geometric/material parameters chosen a priori, the following are based on the built space basis, and only the $z$-functions are updated. If the residual error remains too high, only few new couples are computed to achieve the convergence. Note that this idea has already been used in other works ${ }^{59-62}$ in the framework of nonlinear problems with a time-space decomposition to perform multiparametric analysis. Here, it is extended to the space decomposition in the framework of the composite shell structure.

Several static tests are presented, validating our approach (denoted VS-LD4) and evaluating its efficiency. First, the dedicated approach is assessed by varying the stacking sequences of the laminates. Usual stacking sequences used in aeronautics are addressed. Then, the multiresolution process is carried out for different geometries of the shell. Finally, the possibilities of the approach are illustrated by considering a variation of both the geometry and the number of layers. We pay special attention to the assessment of the out-of-plane stresses that are of relevant importance in the damage phenomenon of such structures. Note that the accuracy of the results is assessed by a comparison with a fourth-order layer-wise model (denoted LD4), referring to the systematic work of Carrera and Carrera's unified formulation. ${ }^{63,64}$

All of the following numerical assessments are based on the configuration proposed in the work of Charrier et al..$^{65}$ It concerns L-angle specimens that are usually used in the junction between different perpendicular panels of the aeronautical structures. It is described as follows.

Geometry: a very thick composite cylindrical shell with $\phi=\pi / 2$. The number of layers (NC) with the stacking sequences and the radius $R$ are precise in each section. All layers have the same thickness.

Boundary conditions: a shell clamped along one straight edge and free on the other sides, subjected to a constant global pressure: $q(\xi)=q_{0}=1 \mathrm{MPa}$. 
TABLE 1 Geometry of the shell

\begin{tabular}{ccccccc} 
& $\boldsymbol{n}$ & No. of Layers & Layer Thickness, $\mathbf{m m}$ & $\phi$ & $\boldsymbol{R}, \mathbf{m m}$ & $\boldsymbol{L}_{\boldsymbol{y}}, \mathbf{m m}$ \\
SP1 & 1 & 16 & 0.262 & $\pi / 2$ & 6.095 & 20 \\
SP2 & 2 & 32 & 0.262 & $\pi / 2$ & 12.19 & 40 \\
SP3 & - & 8 & 0.262 & $\pi / 2$ & 6.095 & 20 \\
\hline
\end{tabular}

Material properties: the T700GC/M21 laminates are considered with the following characteristics: $E_{L}=115 \mathrm{GPa}, E_{T}=8.5 \mathrm{GPa}, G_{L T}=4.5 \mathrm{GPa}$, where $L$ refers to the fiber direction, $T$ refers $G_{T T}=3.0357 \mathrm{GPa}, v_{L T}=0.32, v_{T T}=0.4$, to the transverse direction.

Mesh: The mesh consists of $\left(N_{x}=16\right) \times\left(N_{y}=16\right)$ elements in the $\xi^{1}$ and $\xi^{2}$ directions, respectively. It is considered as a suitable mesh for the present test cases.

Number of dofs: $N d o f_{x y}=3\left(N_{x}+1\right)\left(N_{y}+1\right)$ and $N d o f_{z}=12 \times \alpha N C+3$ are the number of dofs of the two problems associated with $v_{j}^{i}$ and $f_{j}^{i}$, respectively. $\alpha$ is the number of numerical layers per physical layer. Hence, the total number of dofs is $N d o f_{x y}+N d o f_{z}$.

Reference values: LD4 model.

Different stacking sequences (reported in the work of Charrier et $\mathrm{al}^{65}$ ), which can be considered as a large class of lay-up types, are addressed. They are described as follows:

- LU1: any laminate $\left[0^{\circ} / 45^{\circ} / 90^{\circ} /-45^{\circ} / 0^{\circ} / 45^{\circ} /-45^{\circ} / 90^{\circ}\right]$, it has no symmetry;

- LU2: a highly oriented laminate $\left[0_{3}^{\circ} / 45^{\circ} / 90_{2}^{\circ} /-45^{\circ} / 0^{\circ}\right]_{n s}$, usually used to be subjected to high uniaxial tensile loading;

- LU3: a quasi-isotropic laminate $\left[\left(45^{\circ} / 90^{\circ} /-45^{\circ} / 0^{\circ}\right)_{2}\right]_{n s}$, usually used in order to be subjected to complex multiaxial loadings;

- LU4: a highly disoriented laminate $\left[45_{2}^{\circ} / 0^{\circ} /-45_{3}^{\circ} / 90^{\circ} / 45^{\circ}\right]_{n s}$ designed mainly to be subjected to in-plane shear loading, including $75 \%$ of $\pm 45^{\circ}$ plies.

The different geometries of the shell involved in the subsequent examples are summarized in Table 1. In particular, $n$ is given. Each configuration will be denoted LUI-SPJ, where $I \in\{1,2,3,4\}$ and $J \in\{1,2,3\}$.

\subsection{Initial solution}

First, the LU2-SP2 specimen with 32 layers is considered as the initial set of parameters. Eight couples are built to obtain the solution. The distributions of the in-plane functions $v_{i}^{j}(\xi)$ and the thickness functions $f_{i}^{j}(z)$ are given in Figures 3 and 4 . In Figure 3B, $v_{1}^{j}(\xi)$ is associated to the displacement along the radius in the curvilinear coordinate system.
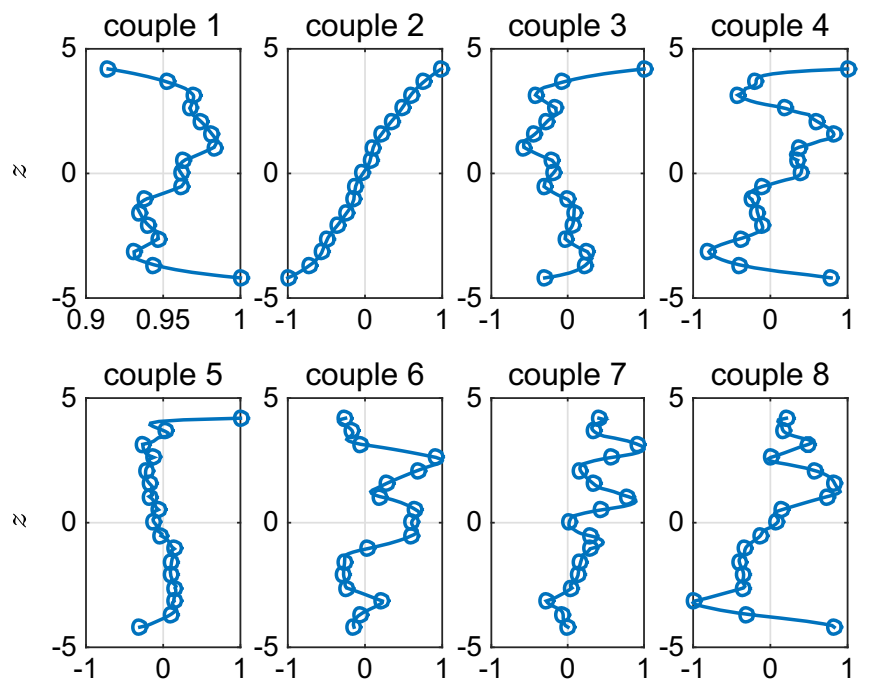

(A)
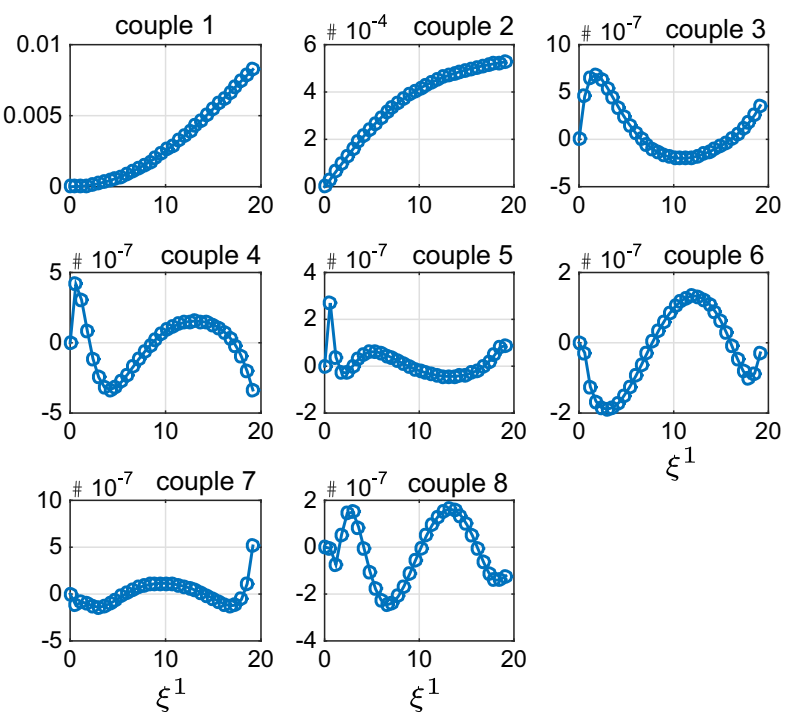

(B)

FIGURE 3 Couples for LU2-SP2. A, $f_{1}^{j}(z)$; B, $v_{1}^{j}\left(\xi^{1}, \xi^{2}=L_{y} / 2\right)$ [Colour figure can be viewed at wileyonlinelibrary.com] 

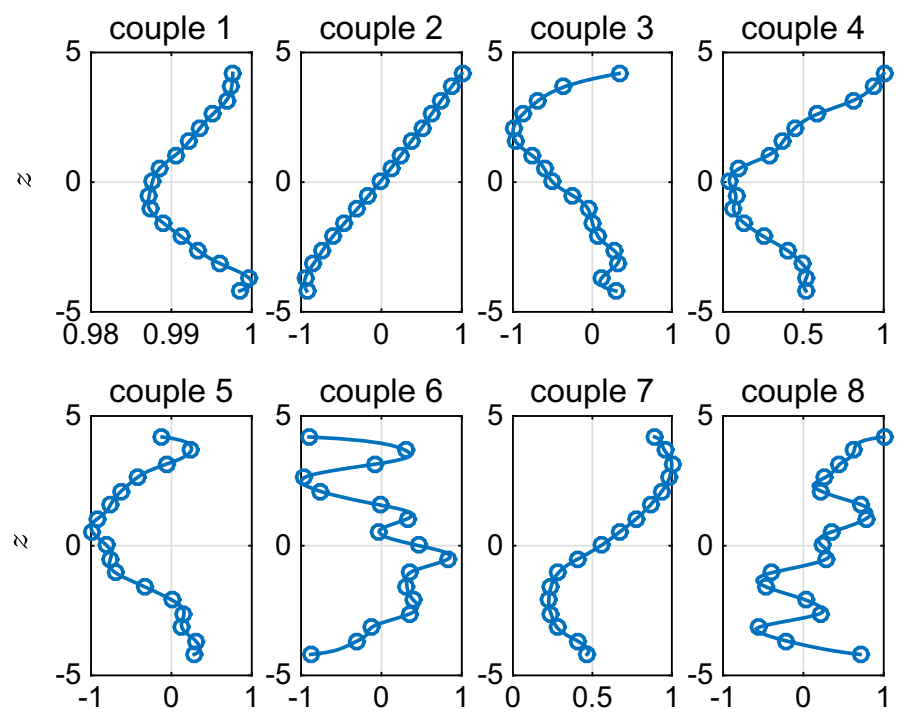

(A)
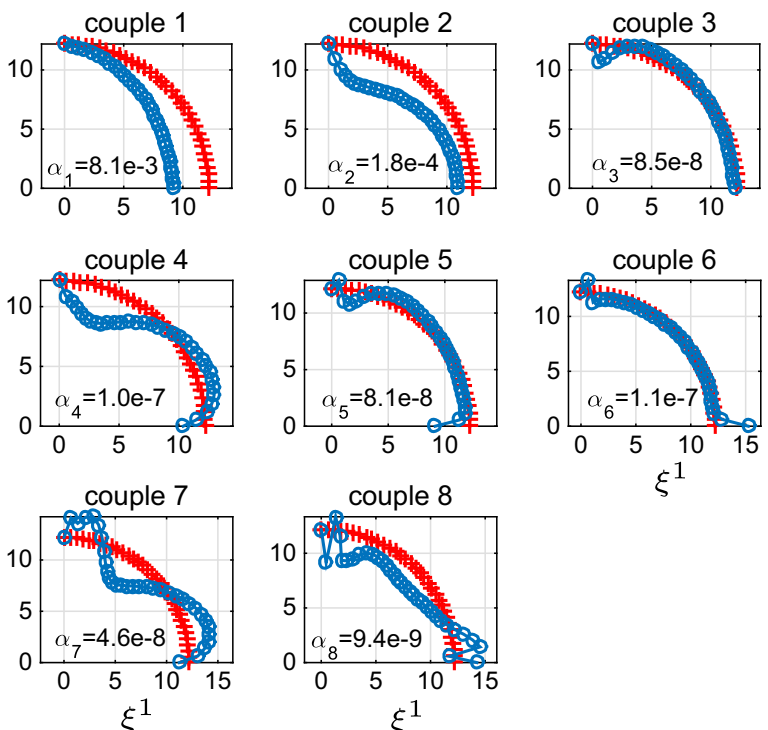

(B)

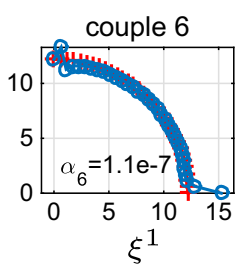

FIGURE 4 Couples for LU2-SP2. A, $f_{3}^{j}(z) ; \mathrm{B}, v_{3}^{j}\left(\xi^{1}, \xi^{2}=L_{y} / 2\right)$ [Colour figure can be viewed at wileyonlinelibrary.com]
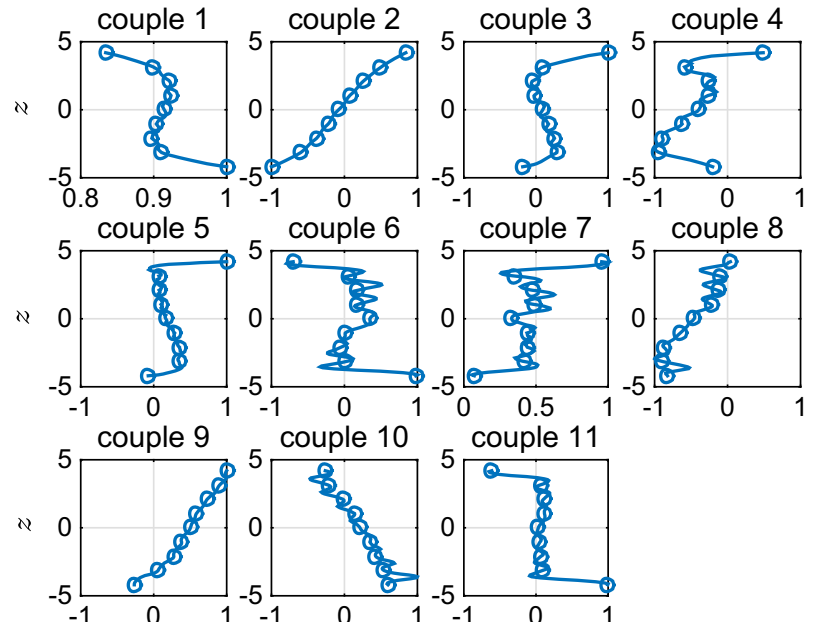

(A)
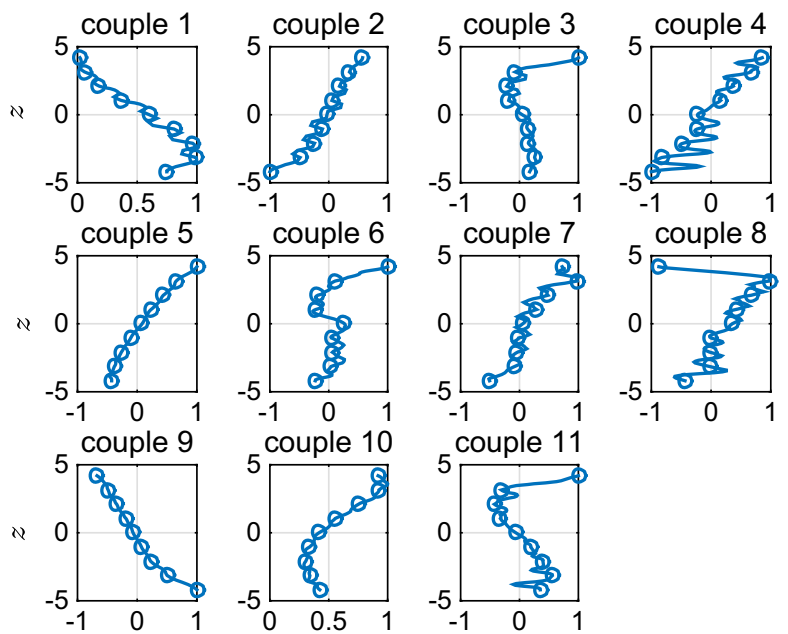

(B)
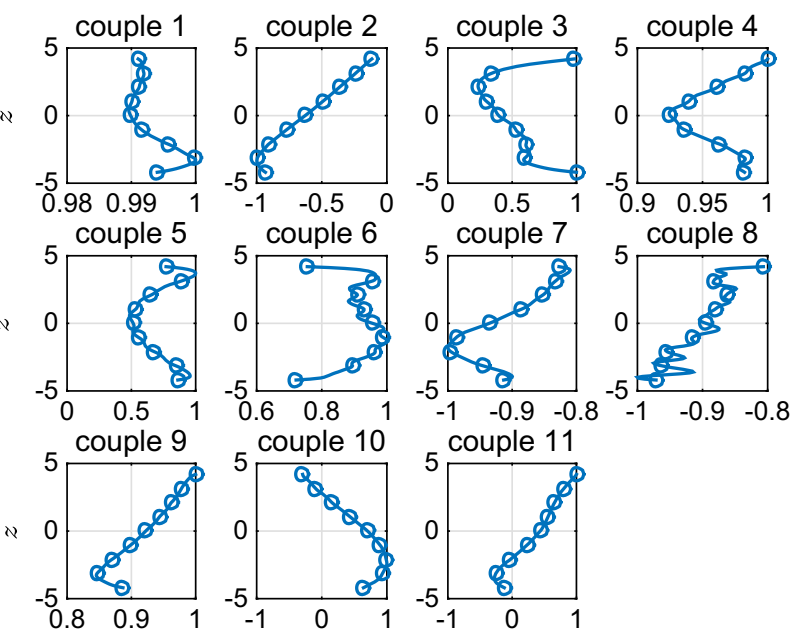

(C)

FIGURE $5 f_{j}^{i}(z)$ LU3-SP2 - multiresolution from couples LU2-SP2. A, $f_{1}^{i}(z) ; \mathrm{B}, f_{2}^{i}(z)$; C, $f_{3}^{i}(z)$ [Colour figure can be viewed at wileyonlinelibrary.com] 
In Figure $4 \mathrm{~B}, v_{3}^{j}(\xi)$ is associated to the transverse displacement. This function is plotted with respect to the undeformed shape of the structure (red cross) in the plane $\xi^{2}=L_{y} / 2$, and the amplification ratio $\alpha_{i}$ is given for all couples, allowing us to compare them against each other. $f_{i}^{j}(z)$ are normalized such that $\max \left|f_{i}^{j}(z)\right|=1$. The two first functions represent the main contributions to the displacement solution. They can be considered as global modes. On the contrary, the following functions involve local corrections that are localized close to the clamped or free edges. For the latter, the distribution of the associated $z$-functions through the thickness is rather complex. Thus, highly refined models are required to capture the variations of the mechanical quantities.

\section{2 | Multiresolution: different lay-up configurations}

Once the eight couples are built for the LU2-SP2 specimen, they are now used for two subsequent configurations, namely, the LU3 and LU4-SP2 shells. The new $z$-functions associated to the LU3-SP2 shell are given in Figure 5. The change of stacks requires the correction of the thickness functions. It should be noted that the two first $f_{3}^{j}(z)$ functions remain quasi-constant and linear. To assess the accuracy of the results, the error rate of the local stresses $\sigma_{11}, \sigma_{13}$, and $\sigma_{33}$ at $\xi^{1}=L_{\xi} / 4, \xi^{2}=L_{y} / 2$ is first evaluated, as it is located in an area with the highest stresses. The results are summarized in Table 2. The calculation of these stresses with the initial functions drives an important error rate. This can be reduced significantly by updating the eight $z$-functions $f_{i}^{j}(z)$. The maximum error rate becomes $3.7 \%$. The accuracy can be improved by enriching the basis with only three functions (error of less than 1.8\%). For further assessment, the distributions of $\sigma_{11}$ and $\sigma_{13}$ through the thickness are compared for the initial basis and the updated version with three new couples in Figure 6. Significant corrections are provided to the initial solution. The accuracy of the results becomes very good with respect to the LD4 model. The complex behavior of the out-of-plane stress is also well described. The continuity of this stress is fulfilled. For this test case, the order of magnitude of the transverse shear and normal stresses

TABLE 2 Multiresolution process from LU2-SP2 $\xi$ basis functions - 32 layers

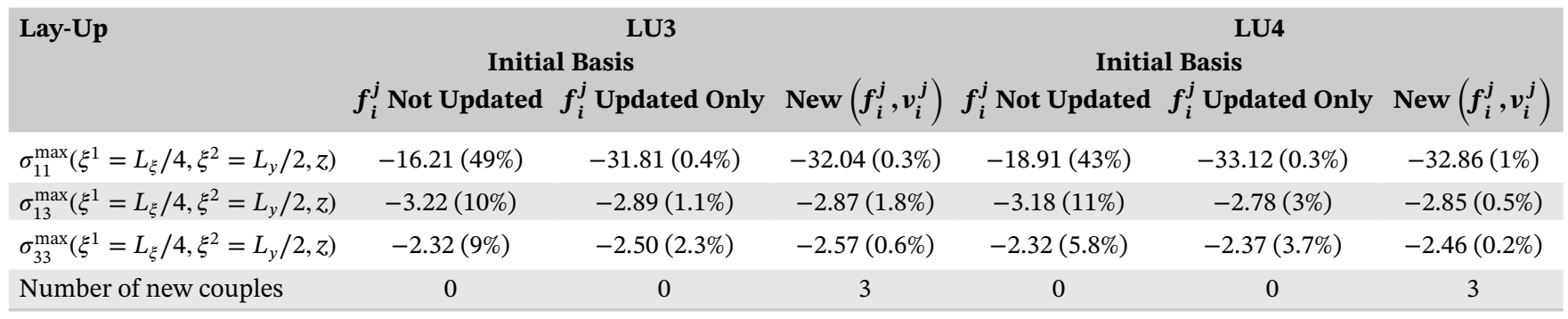
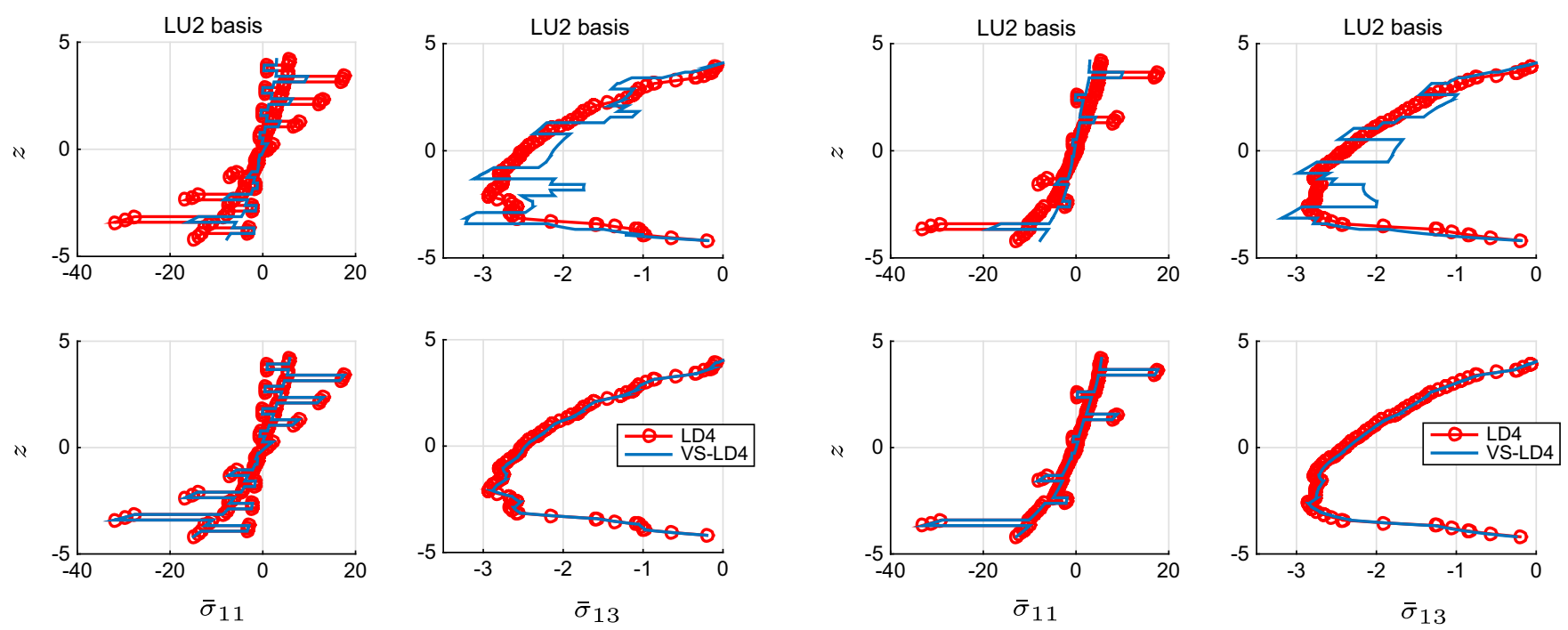

(A)

FIGURE 6 Distribution of $\sigma_{11}\left(\xi^{1}=L_{\xi} / 2, \xi^{2}=L y / 2, z\right)$ and $\sigma_{13}\left(\xi^{1}=L_{\xi} / 2, \xi^{2}=L y / 2, z\right)$ along the thickness - multiresolution process from LU2-SP2 $(\xi)$ basis functions - 32 layers. A, LU3; B, LU4 [Colour figure can be viewed at wileyonlinelibrary.com] 
is the same in each configuration. Note that the maximum values are located at about $z=-e / 4$. To illustrate the capability of the present method to provide quasi-3D results, the distribution of stresses over the plane $\xi^{2}=L_{y} / 2$ is also shown in Figures 7, 8, and 9. The results appear in very good agreement with the LD4 model. Even the local effects, such as the clamped-edge ones, are rather similar. Ignoring this effect, the area where solicitation is most significant is also very well described (around $\xi^{1}=L_{\xi} / 4$ and $z=-e / 4$ ). Finally, despite the strong gap between the initial solution (only the initial functions) and the final solutions (with three new couples) illustrated in Figure 6, a very good accuracy level is achieved with only few new couples. The method can be carried out for a large variety of stacking sequences.

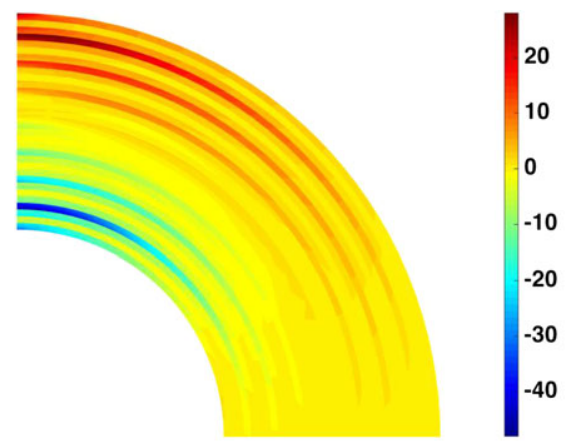

(A)

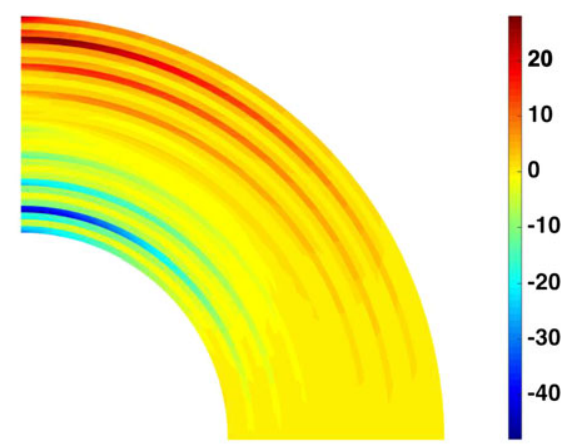

(B)

FIGURE $7 \sigma_{11}\left(\xi^{1}, \xi^{2}=L_{y} / 2, z\right)$ - LU3-SP2 - 32 layers. A, VS-LD4; B, LD4 [Colour figure can be viewed at wileyonlinelibrary.com]

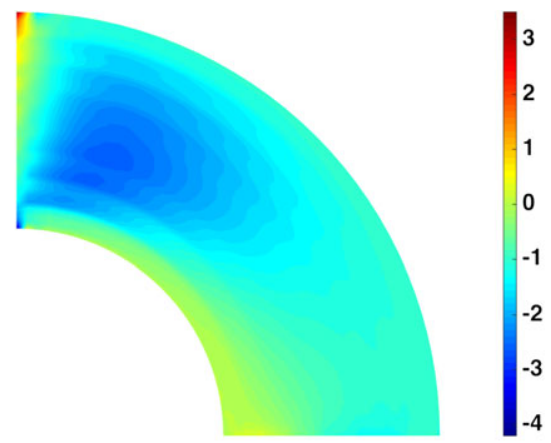

(A)

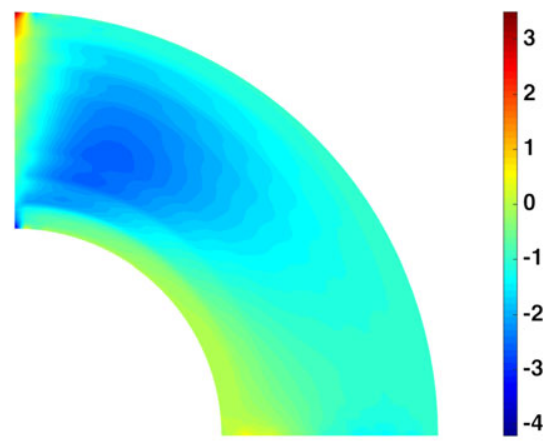

(B)

FIGURE $8 \sigma_{13}\left(\xi^{1}, \xi^{2}=L_{y} / 2, z\right)$ - LU3-SP2 - 32 layers. A, VS-LD4; B, LD4 [Colour figure can be viewed at wileyonlinelibrary.com]

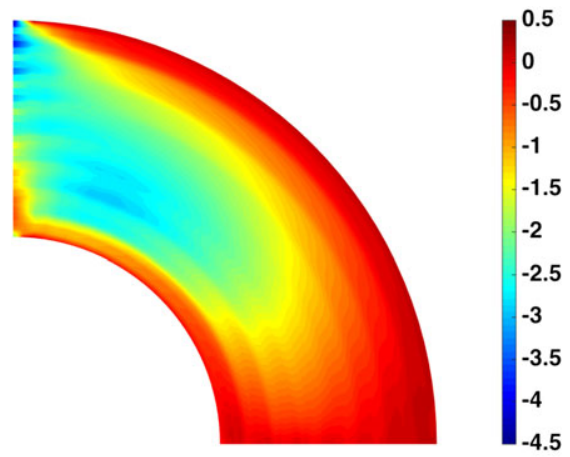

(A)

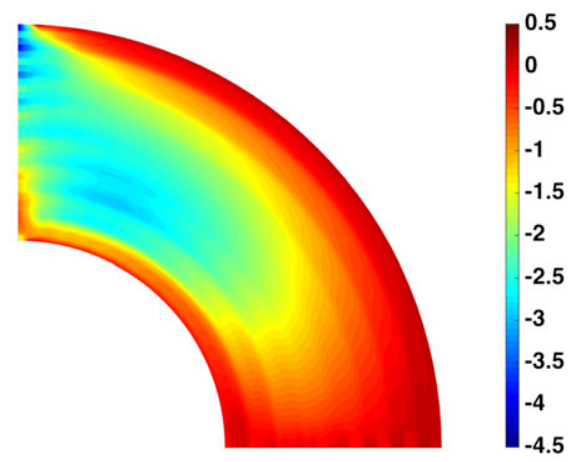

(B)

FIGURE $9 \sigma_{33}\left(\xi^{1}, \xi^{2}=L_{y} / 2, z\right)$ - LU3-SP2 - 32 layers. A, VS-LD4; B, LD4 [Colour figure can be viewed at wileyonlinelibrary.com] 


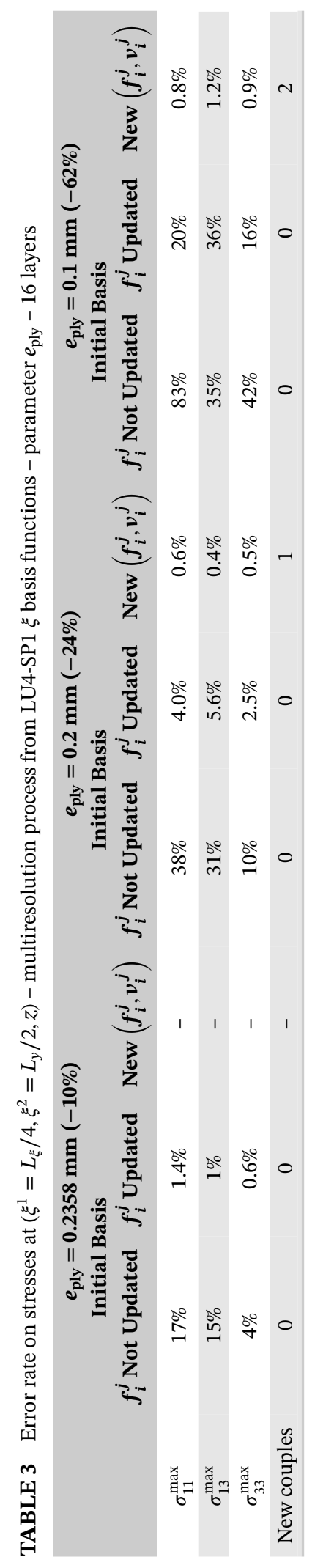




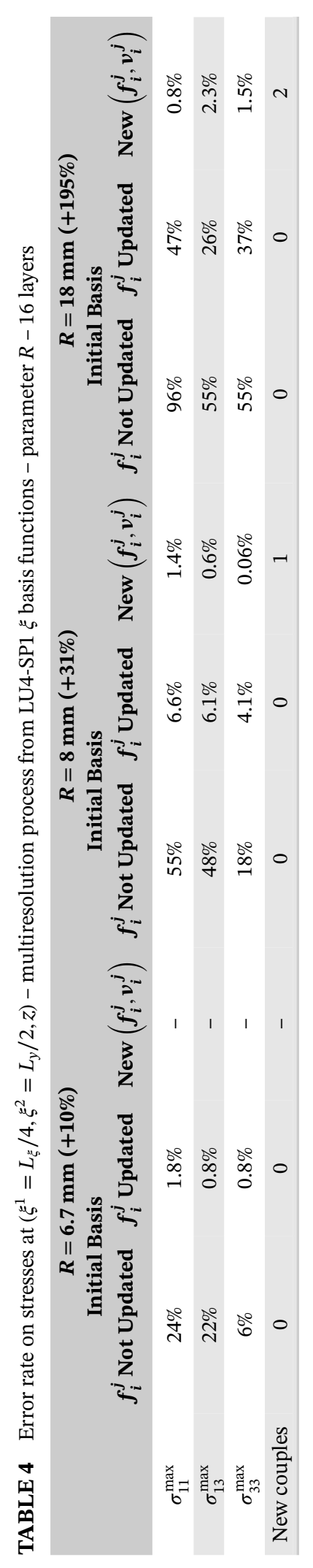




\section{3 | Multiresolution: different geometries}

The same procedure is tested, considering a variation of the geometry. In this study, the involved parameters are the thickness of each layer $e_{\text {ply }}$, the radius of the shell $R$, and the opening angle $\phi$. The initial basis consists of 13 couples for the LU4-SP1 configuration (16 layers). A simple mapping of the 1D domain (for $e_{\text {ply }}$ and $R$ ) or the 2D domain (for $\phi$ ) is achieved for the following computations. The results are given in Tables 3, 4, and 5. As previously, a local error on the stresses is provided. Wide domains of variation of the geometry have been considered. For a variation of $10 \%$ of the parameters, no additional couple is required to obtain an accurate solution; only the $z$-functions have to be updated. Beyond this value, only one or two couples are built despite the high geometric modification. Note that the basis $\xi$-functions seem to be sufficiently rich to represent the changes of the opening angle as the update of the $z$-functions allows us to decrease drastically the error rate (see Table 5).

TABLE 5 Error rate on stresses at $\left(\xi^{1}=L_{\xi} / 4, \xi^{2}=L_{y} / 2, z\right)-$ multiresolution process from LU4-SP1 $\xi$ basis functions - parameter $\phi-16$ layers

\begin{tabular}{|c|c|c|c|c|c|c|}
\hline & \multicolumn{2}{|c|}{$\begin{array}{l}\qquad \phi=\frac{3}{4} \frac{\pi}{2}(-25 \%) \\
\text { Initial Basis }\end{array}$} & \multicolumn{4}{|c|}{$\begin{array}{l}\qquad \phi=\frac{1}{2} \frac{\pi}{2}(-50 \%) \\
\text { Initial Basis }\end{array}$} \\
\hline & $f_{i}^{j}$ Not Updated & $f_{i}^{j}$ Updated Only & $\operatorname{New}\left(f_{i}^{j}, v_{i}^{j}\right)$ & $f_{i}^{j}$ Not Updated & $f_{i}^{j}$ Updated Only & $\operatorname{New}\left(f_{i}^{j}, v_{i}^{j}\right)$ \\
\hline$\sigma_{11}^{\max }$ & $122 \%$ & $0.6 \%$ & - & $880 \%$ & $0.8 \%$ & $0.7 \%$ \\
\hline$\sigma_{13}^{\max }$ & $97 \%$ & $0.9 \%$ & - & $447 \%$ & $2.7 \%$ & $1.7 \%$ \\
\hline$\sigma_{33}^{\max }$ & $34 \%$ & $0.3 \%$ & - & $30 \%$ & $0.1 \%$ & $0.9 \%$ \\
\hline New couples & 0 & 0 & - & 0 & 0 & 1 \\
\hline
\end{tabular}
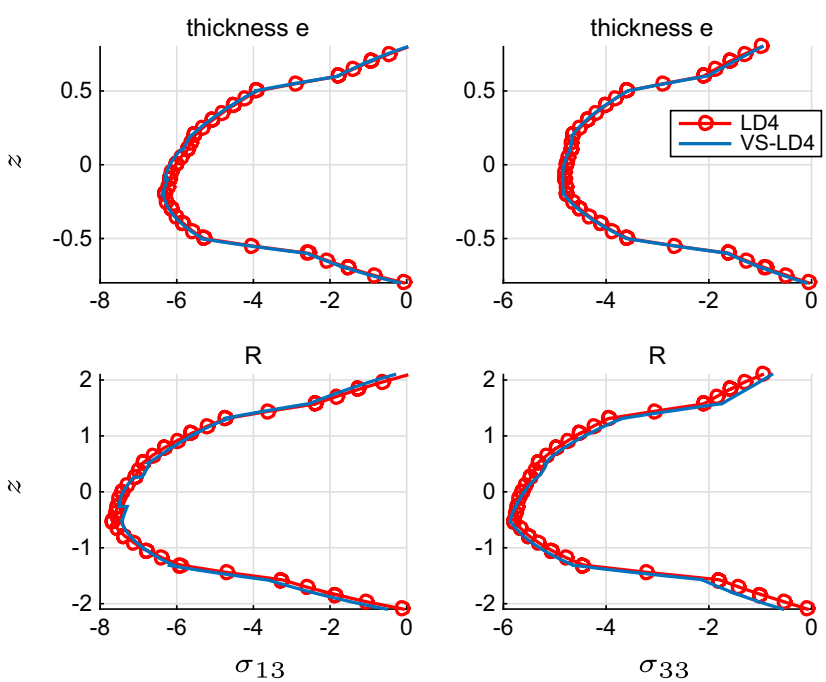

FIGURE 10 Distribution of $\sigma_{13}\left(\xi^{1}=L_{\xi} / 2, \xi^{2}=L y / 2, z\right)$ and $\sigma_{33}\left(\xi^{1}=L_{\xi} / 2, \xi^{2}=L y / 2, z\right)$ along the thickness - multiresolution process from LU4-SP1 basis $\xi$-functions - parameter $e_{\text {ply }}, R-16$ layers [Colour figure can be viewed at wileyonlinelibrary.com]

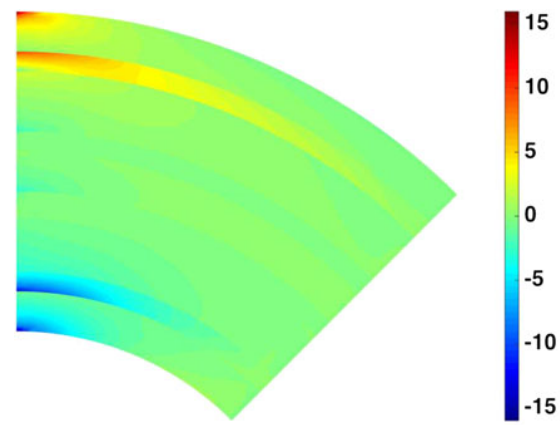

(A)

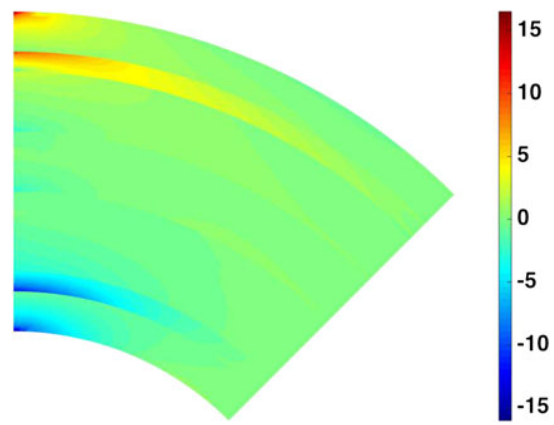

(B)

FIGURE $11 \sigma_{11}\left(\xi^{1}, \xi^{2}=L_{y} / 2, z\right)$ - LU4-SP1 - 16 layers. A, VS-LD4; B, LD4 [Colour figure can be viewed at wileyonlinelibrary.com] 


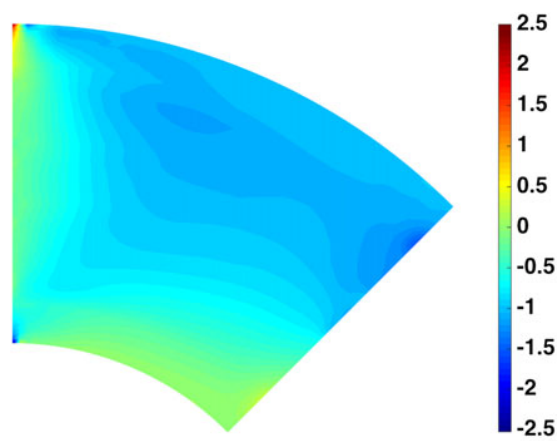

(A)

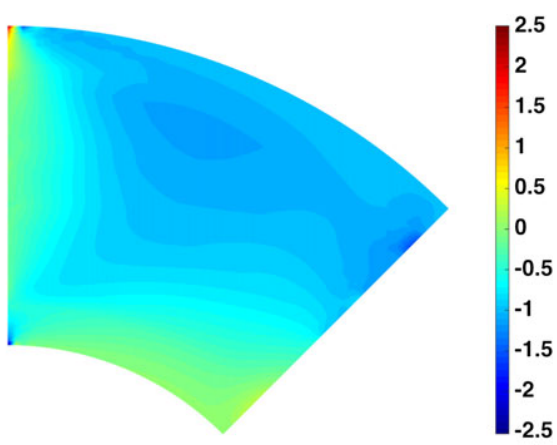

(B)

FIGURE $12 \sigma_{13}\left(\xi^{1}, \xi^{2}=L_{y} / 2, z\right)$ - LU4-SP1 - 16 layers. A, VS-LD4; B, LD4 [Colour figure can be viewed at wileyonlinelibrary.com]

For further comparison, the distribution of the out-of-plane stresses through the thickness is presented in Figure 10 for $e_{\mathrm{ply}}=0.1 \mathrm{~mm}$ and $R=18 \mathrm{~mm}$. It can be shown that the results are in excellent agreement with the reference LD4 solution. The top/bottom conditions are fulfilled. Then, the distribution of $\sigma_{11}$ and $\sigma_{13}$ over the plane $\xi^{2}=L_{y} / 2$ for $\phi=\frac{\pi}{4}$ is given in Figures 11 and 12. It can be also inferred from these figures that the accuracy of the results is very satisfactory. The localization of the in-plane stresses is well captured near the clamped edge.

\section{4 | Multiresolution: different geometries with different numbers of layers}

In this section, the multiresolution process is performed with different numbers of layers and different total thicknesses. We take advantage of the separation of the solution between the in-plane and out-of-plane functions. Thus, using the

TABLE 6 Error rate on stresses at $\left(\xi^{1}=L_{\xi} / 4, \xi^{2}=L_{y} / 2, z\right)-$ multiresolution process from LU4-SP1 $\xi$ basis functions - different lay-ups

\begin{tabular}{lcccc} 
& \multicolumn{2}{c}{ 32 Layers LU2-SP2 } & \multicolumn{2}{c}{ 8 Layers LU1-SP3 } \\
& $\boldsymbol{f}_{\boldsymbol{i}}^{\boldsymbol{j}}$ Updated & $\mathbf{N e w}\left(\boldsymbol{f}_{\boldsymbol{i}}^{\boldsymbol{j}}, \boldsymbol{v}_{\boldsymbol{i}}^{\boldsymbol{j}}\right)$ & $\boldsymbol{f}_{\boldsymbol{i}}^{\boldsymbol{j}}$ Updated & New $\left(\boldsymbol{f}_{\boldsymbol{i}}^{\boldsymbol{j}}, \boldsymbol{v}_{\boldsymbol{i}}^{\boldsymbol{j}}\right)$ \\
\hline$\sigma_{11}^{\max }$ & $1.2 \%$ & $0.7 \%$ & $15 \%$ & $0.9 \%$ \\
$\sigma_{13}^{\max }$ & $5.2 \%$ & $0.04 \%$ & $19 \%$ & $1.1 \%$ \\
$\sigma_{33}^{\max }$ & $3.7 \%$ & $0.6 \%$ & $9 \%$ & $0.1 \%$ \\
New couples & 0 & 2 & 0 & 2 \\
\hline
\end{tabular}
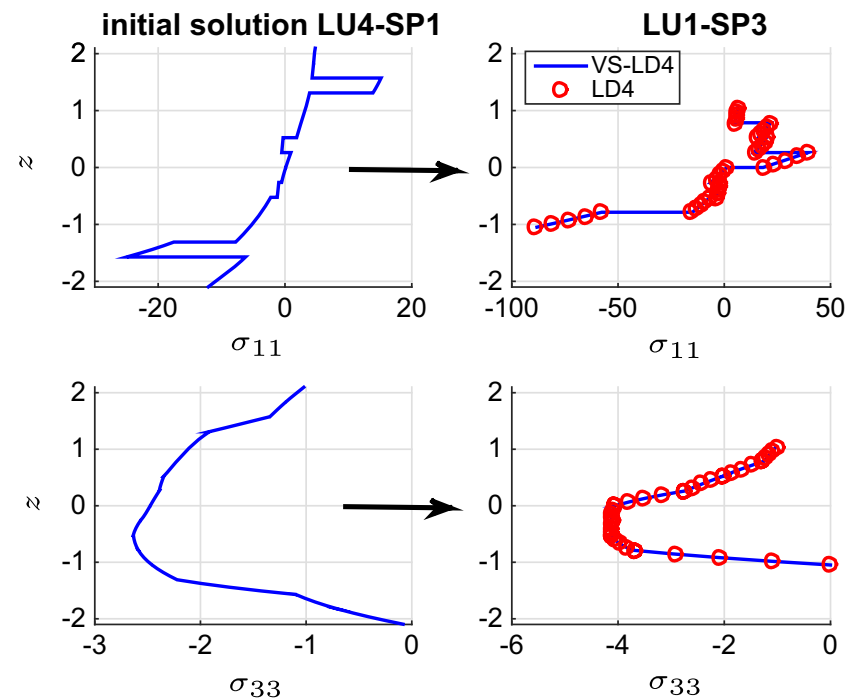
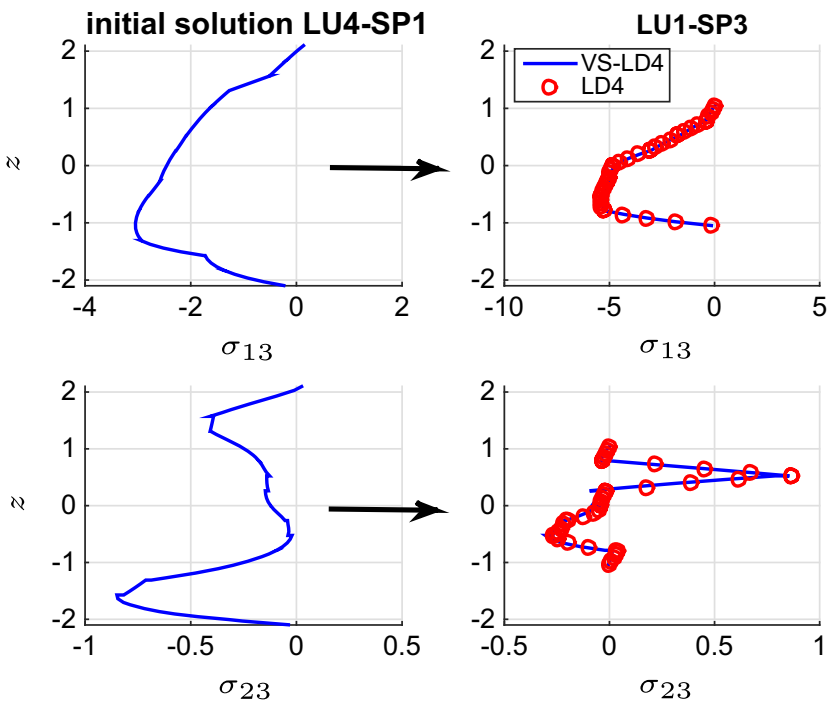

FIGURE 13 Distribution of $\sigma_{11}\left(\xi^{1}=L_{\xi} / 4, \xi^{2}=L y / 2, z\right), \sigma_{13}\left(\xi^{1}=L_{\xi} / 4, \xi^{2}=L y / 2, z\right)$, and $\sigma_{33}\left(\xi^{1}=L_{\xi} / 2, \xi^{2}=L y / 2, z\right)$ along the thickness multiresolution process from LU4-SP1 $\xi$ basis functions - geometry - 16 layers [Colour figure can be viewed at wileyonlinelibrary.com] 
13 in-plane functions computed for the LU4-SP1 shell (16 plies), the solution can be built for the LU2-SP2 (32 plies; $e=8.384 \mathrm{~mm}$ ) and LU1-SP3 (eight plies; $e=2.096 \mathrm{~mm}$ ) configurations. Table 6 shows that two new couples allow us to have an error rate of less than $1.1 \%$. The comparison of the initial solution (LU4-SP1) and the final solution (LU1-SP3 [eight plies]) is given in Figure 13. The solutions are deeply different. This figure shows also that the distribution of the in-plane and out-of-plane stresses through the thickness is very accurate when compared with the LD4 model.

This test case allows us to show the possibilities of the method to address many shell configurations.

\section{6 | CONCLUSION}

In this paper, PGD is advantageously performed in the framework of the multiresolution process for modeling composite shell structures. Indeed, the in-plane functions built for one fixed set of parameters can be reused for the subsequent configurations, reducing the computational cost to a 1D problem. If needed, only few new couples are computed. The performance of the method is illustrated for a wide type of shell structures. The changes of stacking sequences, geometry (radius, thickness, and open angle), and number of layers have been addressed. The good accuracy of the results is shown by a comparison with a quasi-3D layer-wise reference solution. Thus, the potential of the approach seems to be very interesting in the framework of multiparametric modeling of composite structures.

\section{ORCID}

P. Vidal (iD https://orcid.org/0000-0002-9634-4443

\section{REFERENCES}

1. Dau F, Polit O, Touratier M. An efficient $\mathrm{C}^{1}$ finite element with continuity requirements for multilayered/sandwich shell structures. Comput Struct. 2004;82:1889-1899.

2. Zienkiewicz OC, Taylor RL. The Finite Element Method: The Basis. 5th ed. Oxford, UK: Butterworth-Heinemann; 2000.

3. Vidal P, D'Ottavio M, Thaïer MB, Polit O. An efficient finite shell element for the static response of piezoelectric laminates. J Intell Material Syst Struct. 2011;7(22):671-690.

4. Reddy JN. Mechanics of Laminated Composite Plates and Shells: Theory and Analysis. Boca Raton, FL: CRC Press; 2004.

5. Leissa AW. Vibration of shells. 1973. Nasa Technical Report. NASA SP-288.

6. Rao KP. Rectangular laminated anisotropic shallow thin shell finite element. Comput Methods Appl Mech Eng. 1978;15:13-33.

7. Jeyachandrabose C, Kirkhope J. Explicit formulation of two anisotropic, triangular, thin, shallow shell elements. Comput Struct. 1987;25:415-436.

8. Qatu M, Leissa A. Bending analysis of laminated plates and shells by different methods. Comput Struct. 1994;52:529-539.

9. Reddy JN. Bending of laminated anisotropic shells by a shear deformable finite element. Fibre Sci Technol. 1982;17:9-24.

10. Chakravorty D, Bandyopadhyay JN, Sinha PK. Finite element free vibration analysis of doubly curved laminated composite shells. J Sound Vib. 1996;191:491-504.

11. Hossain SJ, Sinha PK, Sheikh AH. A finite element formulation for the analysis of laminated composite shells. Comput Struct. 2004;82:1623-1638.

12. Asadi E, Wang W, Qatu MS. Static and vibration analyses of thick deep laminated cylindrical shells using 3D and various shear deformation theories. Compos Struct. 2012;94(2):494-500.

13. Reddy JN, Liu CF. A higher-order shear deformation theory of laminated elastic shells. Int J Eng Sci. 1985;23:319-330.

14. Balah M, Al-Ghamedy HN. Finite element formulation of a third order laminated finite rotation shell element. Comput Struct. 2002;80:1975-1990.

15. Sgambitterra G, Adumitroaie A, Barbero EJ, Tessler A. A robust three-node shell element for laminated composites with matrix damage. Compos Part B Eng. 2011;42:41-50.

16. Kant T, Menon MP. Estimation of interlaminar stresses in fibre reinforced composite cylindrical shells. Comput Struct. 1991;38:131-147.

17. Carrera E. Theories and finite elements for multilayered, anisotropic, composite plates and shells. Arch Comput Methods Eng. 2002;9:87-140.

18. Carrera E. Historical review of zig-zag theories for multilayered plates and shells. Appl Mech Rev. 2003;56(3):287-308.

19. Cinefra M, Carrera E. Shell finite elements with different through-the-thickness kinematics for the linear analysis of cylindrical multilayered structures. Int J Numer Methods Eng. 2013;93(2):160-182.

20. Bhaskar K, Varadan TK. A higher-order theory for bending analysis of laminated shells of revolution. Comput Struct. 1991;40(4):815-819.

21. Jing H-S, Tzeng K-G. Refined shear deformation theory of laminated shells. AIAA J. 1993;31(4):765-773.

22. Brank B. On composite shell models with a piecewise linear warping function. Compos Struct. 2003;59:163-171. 
23. Ganapathi M, Patel BP, Patel HG, Pawargi DS. Vibration analysis of laminated cross-ply oval cylindrical shells. J Sound Vibr. 2003;262:65-86.

24. Botello S, Oñate E, Canet JM. A layer-wise triangle for analysis of laminated composite plates and shells. Comput Struct. 1999;70:635-646.

25. Zinno R, Barbero EJ. A three-dimensional layer-wise constant shear element for general anisotropic shell-type structures. Int J Num Methods Eng. 1994;37:2445-2470.

26. Seide P, Chaudhuri RA. Triangular finite element for analysis of thick laminated shells. Int J Num Methods Eng. 1987;24(8):1563-1579.

27. Basar Y, Ding Y. Interlaminar stress analysis of composites: layer-wise shell finite elements including transverse strains. Compos Eng. 1995;5(5):485-499.

28. Grigolyuk EI, Kulikov GM. General direction of development of the theory of multilayered shells. Mech Compos Mater. 1988;24: 231-241.

29. Kulikov GM, Plotnikova SV. Advanced formulation for laminated composite shells: 3D stress analysis and rigid-body motions. Compos Struct. 2013;95:236-246

30. Soldatos KP, Timarci T. A unified formulation of laminated composite, shear deformable, five-degrees-of-freedom cylindrical shell theories. Compos Struct. 1993;25,(1-4):165-171.

31. Yasin MY, Kapuria S. An efficient layerwise finite element for shallow composite and sandwich shells. Compos Struct. 2013;98:202-214.

32. Cho M, Kim KO, Kim MH. Efficient higher-order shell theory for laminated composites. Compos Struct. 1996;34(2):197-212.

33. Shariyat M. Non-linear dynamic thermo-mechanical buckling analysis of the imperfect laminated and sandwich cylindrical shells based on a global-local theory inherently suitable for non-linear analyses. Int J Non-Linear Mech. 2011;46:253-271.

34. Yasin MY, Kapuria S. An efficient layerwise finite element for shallow composite and sandwich shells. Compos Struct. 2013;98:202-214.

35. Shu X-P. Refined theory of laminated shells with higher order transverse shear deformation. Int J Solids Struct. 1997;34(6):673-683.

36. Zhen W, Wanji C. A global-local higher order theory for multilayered shells and the analysis of laminated cylindrical shell panels. Compos Struct. 2008;84(4):350-361.

37. Icardi U, Ferrero L. Multilayered shell model with variable representation of displacements across the thickness. Compos Part B Eng. 2011;42:18-26.

38. Carrera E, Pagani A, Valvano S. Shell elements with through-the-thickness variable kinematics for the analysis of laminated composite and sandwich structures. Compos Part B Eng. 2017;111:294-314.

39. Kapania RK. A review on the analysis of laminated shells. J Pres Vessel Technol. 1989;111:88-96.

40. Noor AK, Burton WS. Assessment of computational models for multilayered composite shells. Appl Mech Rev. 1990;43(4):67-97.

41. Gilewski W, Radwańska M. A survey of finite element models for the analysis of moderately thick shells. Finite Elem Anal Des. 1991;9:1-21.

42. Yang HTY, Saigal S, Masud A, Kapania RK. Survey of recent shell finite element. Int J Numer Methods Eng. 2000;47:101-127.

43. Carrera E. Theories and finite elements for multilayered, anisotropic, composite plates and shells. Arch Comput Methods Eng. 2002;9(2):87-140.

44. Reddy JN, Arciniega RA. Shear deformation plate and shell theories: from Stavsky to present. Mech Adv Mater Struct. 2004;11:535-582.

45. Hohe J, Librescu L. Advances in the structural modeling of elastic sandwich panels. Mech Adv Mater Struct. 2004;11(4-5):395-424.

46. Qatu MS, Asadi E, Wang W. Review of recent literature on static analyses of composite shells: 2000-2010. Open J Compos Mater. 2012;2:61-86.

47. Caliri MF Jr, Ferreira AJ, Tita V. A review on plate and shell theories for laminated and sandwich structures highlighting the finite element method. Compos Struct. 2016;156:63-77.

48. Chinesta F, Ammar A, Leygue A, Keunings R. An overview of the proper generalized decomposition with applications in computational rheology. J Non-Newtonian Fluid Mech. 2011;166(11):578-592.

49. Chinesta F, Ammar A, Cueto E. Recent advances and new challenges in the use of the proper generalized decomposition for solving multidimensional models. Archives Computat Methods Eng. 2010;17(4):327-350.

50. Ammar A, Mokdad B, Chinesta F, Keunings R. A new family of solvers for some classes of multidimensional partial differential equations encountered in kinetic theory modeling of complex fluids. J Non-Newtonian Fluid Mech. 2006;139:153-176.

51. Savoia M, Reddy JN. A variational approach to three-dimensional elasticity solutions of laminated composite plates. $J$ Appl Mech. 1992;59:S166-S175.

52. Bognet B, Bordeu F, Chinesta F, Leygue A, Poitou A. Advanced simulation of models defined in plate geometries: 3D solutions with 2D computational complexity. Comput Methods Appl Mech Eng. 2011;201-204:1-12.

53. Vidal P, Gallimard L, Polit O. Assessment of a composite beam finite element based on the proper generalized decomposition. Compos Struct. 2012;94(5):1900-1910.

54. Vidal P, Gallimard L, Polit O. Proper generalized decomposition and layer-wise approach for the modeling of composite plate structures. Int J Solids Struct. 2013;50(14-15):2239-2250.

55. Vidal P, Gallimard L, Polit O. Shell finite element based on the proper generalized decomposition for the modeling of cylindrical composite structures. Comput Struct. 2014;132:1-11.

56. Bussy P, Rougée P, Vauchez P. The large time increment method for numerical simulation of metal forming processes. In: Proceedings of the International Conference on Numerical Methods in Engineering: Theory and Applications (NUMETA); 1990; Swansea, UK.

57. Boisse PH, Bussy P, Ladeveze P. A new approach in non-linear mechanics: the large time increment method. Int J Numer Methods Eng. 1990;29:647-663.

58. Bernadou M. Finite Element Methods for Thin Shell Problems. Chichester, UK: Wiley; 1996. 
59. Boucard PA, Ladevèze P. A multiple solution method for non-linear structural mechanics. Mech Eng. 1999;50(5):317-328.

60. Allix O, Vidal P. A new multi-solution approach suitable for structural identification problems. Comput Methods Appl Mech Eng. 2002;191(25-26):2727-2758.

61. Heyberger C, Boucard PA, Néron D. Multiparametric analysis within the proper generalized decomposition framework. Comput Mech. 2011;49:277-289.

62. Néron D, Boucard P-A, Relun N. Time-space PGD for the rapid solution of 3D nonlinear parametrized problems in the many-query context. Int J Numer Methods Eng. 2015;103:275-292.

63. Carrera E, Demasi L. Classical and advanced multilayered plate elements based upon PVD and RMVT. Part 1: derivation of finite element matrices. Int J Numer Methods Eng. 2002;55:191-231.

64. Carrera E, Demasi L. Classical and advanced multilayered plate elements based upon PVD and RMVT. Part 2: numerical implementations. Int J Numer Methods Eng. 2002;55:253-291.

65. Charrier JS, Laurin F, Carrere N, Mahdi S. Determination of the out-of-plane tensile strength using four-point bending tests on laminated L-angle specimens with different stacking sequences and total thicknesses. Compos Part A Appl Sci Manuf. 2016;81:243-253. 